\title{
MOLECULAR COMMUNICATION OPTIONS FOR LONG RANGE NANONETWORKS
}

\section{Autor: Lluís Parcerisa Giné}

Advisor: lan F. Akyildiz

UPC Tutor: Josep Solé Pareta 
This master thesis was realized from September 2008 to July 2009 at the Broadband Wireless Networking (BWN) Laboratory, School of Electrical and Computer Engineering, Georgia Institute of Technology, 250 14th Street, Atlanta, GA 30332, USA.

Ta my family Artur, Núria and Agnès and ta Vialeta.

\section{I wauld like ta thank}

Massimiliana Pieralean, Jasep M. Jarnet and Maria Gregari far their support and critical camments aleaut the present wark.

Finally, my gratitude ta

Jan F. Akyildiz, Jasep Salé and Eduard Alarcán for making this praject passille. 


\section{CONTENTS}

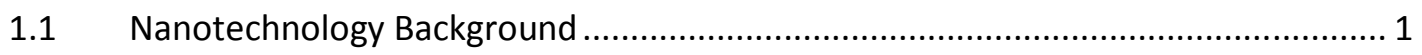

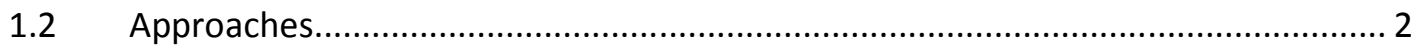

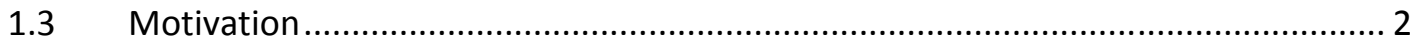

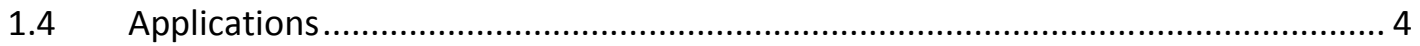

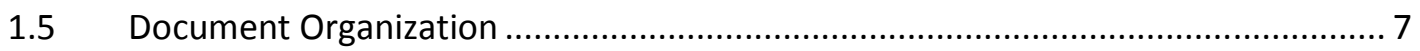

WIRELESS OPTIONS

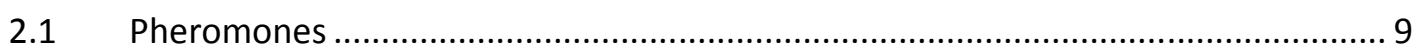

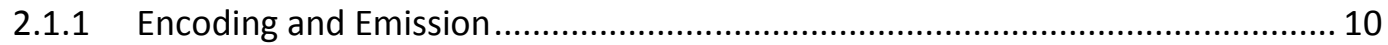

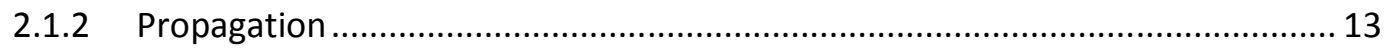

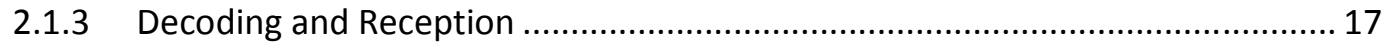

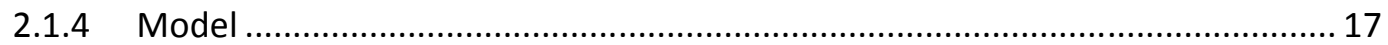

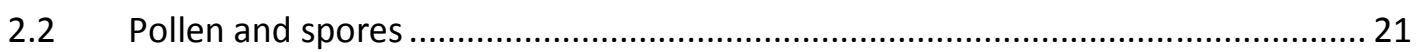

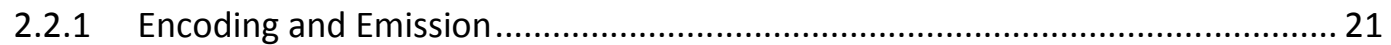

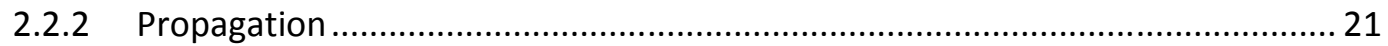

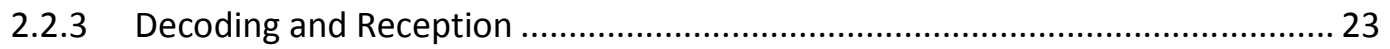

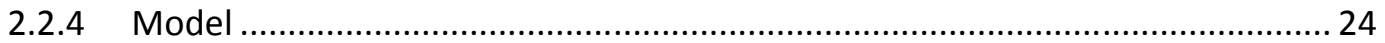

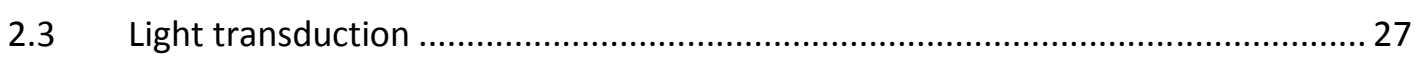

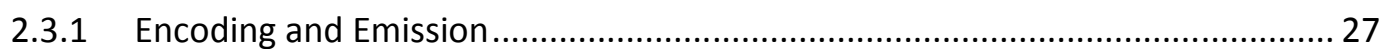

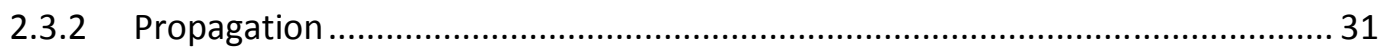

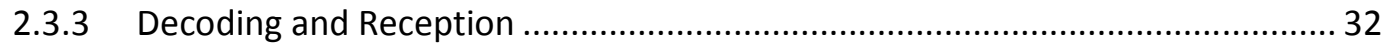

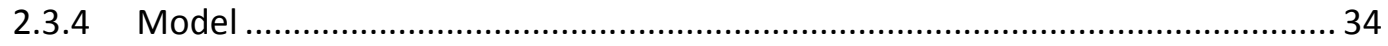

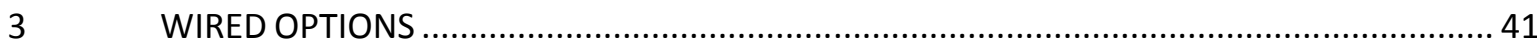

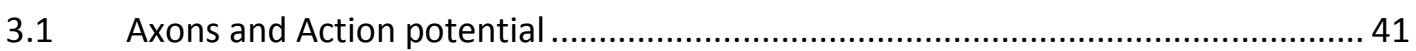

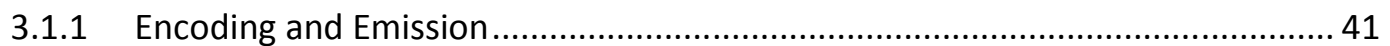

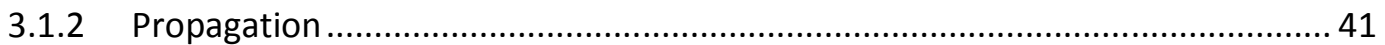




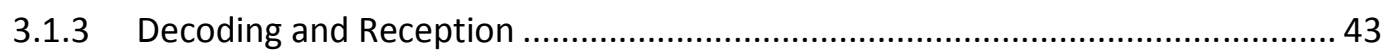

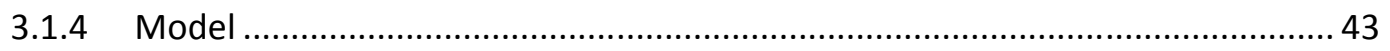

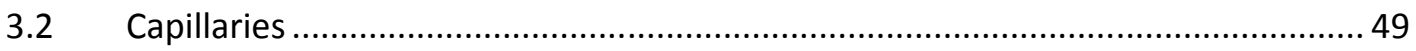

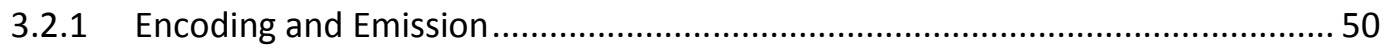

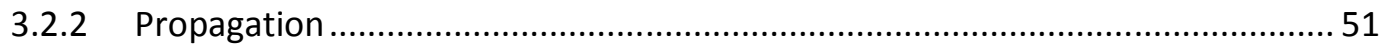

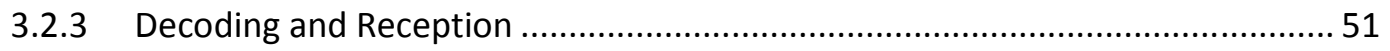

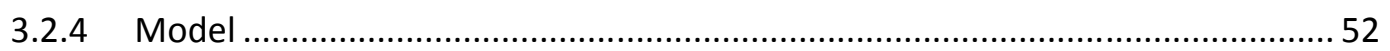

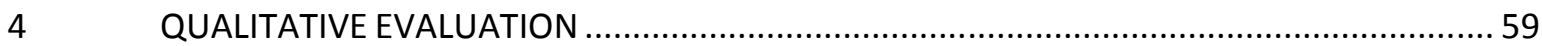

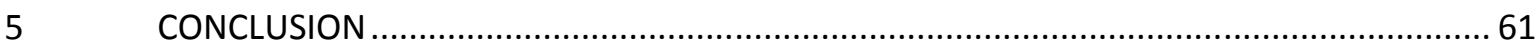

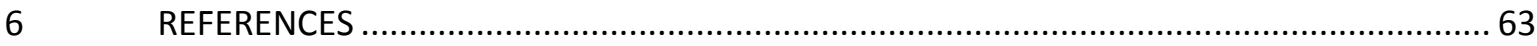




\section{INTRODUCTION}

\subsection{NANOTECHNOLOGY BACKGROUND}

The birth of nanotechnology is often associated with the talk given by Nobel Prize winner Richard Feynman entitled "There's Plenty of Room at the Bottom". In this talk, Feynman discusses the possibilities of what is now commonly referred to as nanotechnology and how its advancement could potentially generate an enormous number of technical applications.

Nanotechnology comprises technological developments on the nanometer scale, usually on the order of 0.1 to $100 \mathrm{~nm}$. For a perspective of this scale at the atomic level, a hydrogen atom's diameter is on the order of an Ångström $(1 \AA=0.1 \mathrm{~nm})$. Thus, ten hydrogen atoms laid side by side would measure a distance of about $1 \mathrm{~nm}$ across. Nanotechnology is necessarily a multidisciplinary field which encompasses and draws from the knowledge of several diverse technological fields of study including chemistry, physics, molecular biology, material science, computer science, and engineering.

Nowadays many technological fields are experiencing one major revolution, coming from the possibility of manipulating and assembling objects at the nanometer scale. In particular, one of the fields where nanotechnologies are expected to have a major impact is electronics, whereby the possibility of working at the atomic scale opens up new possibilities for building faster, smaller, and cheaper integrated circuits. However, when building nanoscale devices, we implicitly come up with the problem of interconnecting them into a purposeful system, able to perform complex functions through the proper interconnection (by means of information transmission) of simple elements.

Advances in the field of nanotechnology have expanded the breadth of potential applications tremendously in recent years. The nanotechnology research and development (R\&D) areas have been growing rapidly throughout the world. The nanotechnology R\&D investment reported by government organizations around the world has increased from approximately $\$ 432$ million in 1997 to about $\$ 3$ billion in 2003 alone. At least 30 countries have initiated national activities in this field. Although nanotechnology is currently still considered to be in the precompetitive stage, the worldwide annual industrial production in the nanotechnology sectors is estimated to exceed \$1 trillion in 10 to 15 years, which would require about 2 million nanotechnology workers. 


\subsection{APPROACHES}

There are three different approaches for the development of nano-machines, namely the top-down, the bottom-up and the bio-hybrid [1].

- In the top-down approach, the existing electronic components and devices are scaled down from the micro domain to the nano domain by means of conventional techniques, such as lithography for silicon-based transistors. However, in the nano-scale, electrons tend to be constrained in such a limited space that current well-established laws are not suitable to completely describe the nano-components behavior. For this, the design of nano-electronic devices should take into account the quantum effects [58].

- In the bottom-up approach, the design of nano-machines is realized from molecular components, which chemically assemble themselves by principles of molecular recognition, molecule by molecule [40] [21]. The most promising technique following this approach is the construction of quantum dots (also known as nanocrystals), which are semiconductor materials at atomic level. However, the fabrication of quantum dots is still a challenging issue [59], and their toxicity makes this option infeasible for medical applications [33], [34]. In this section several techniques are being developed, e.g. the analysis of carbon nano-ribbons properties in [37].

- A third approach, called bio-hybrid, has been proposed for the development of nano-machines. In this case, biological elements can be directly used as building material or as patterns to be replicated with synthesized components [1].

\subsection{Motivation}

Nanotechnology enables the miniaturization and fabrication of devices in a scale ranging from 1 to 100 nanometers. At this scale, a nano-machine can be considered the most basic functional unit. Nano-machines are tiny components consisting of a set of molecules which are able to perform very simple computation, sensing and/or actuation tasks [70]. 
Nano-machines can be interconnected to execute collaborative tasks in a distributed manner. The resulting nanonetworks are envisaged to expand the capabilities and applications of single nano-machines. Communication between nano-machines can be performed through classical methods or by means of molecular communication.

Classical communication techniques (e.g., electromagnetic fields, optical or acoustic waves), cannot be applied to nano-scale networks by merely reducing conventional networks dimensions. The complexity and size of existing transceivers as well as their high power consumption are significant drawbacks that the aforementioned techniques present at nanometer dimensions [37]. As an example, the influence of the quantum effect at the nano-scale limit the applicability of conventional network paradigms based on classical electronics [32]. The main alternative to overcome these disadvantages and still use electromagnetic waves as a supporting carrier for information transmission is the use of novel materials implicitly lying in the nano-domain. For example, a nano-patch antenna based on graphene ribbons is proposed in [37].

The concept of molecular communications has been introduced in the recent years [1]. Following the bio-hybrid approach, molecular communication is inspired by the communication mechanisms that naturally occur between living cells, and it is defined as the transmission of information using molecules.

Bio-inspired molecular communication approach is envisaged to govern the development of future nanonetwork communications. First of all, the natural size of biological elements eliminates the need of downscaling micro components because molecules or biological cells, which can be used as building blocks, are already in the nanometer scale. Biocompatibility provided to the system by molecular communication is another advantage, allowing a more direct interaction with medical applications. Finally, the energy consumption is extremely low compared to semiconductor based networks. According to [70], a single molecular reaction, which may represent multiple computations, consumes 10,000 times less energy than a microelectronic transistor.

Molecular communication takes place amongst living cells via direct contact (juxtacrine signaling), without contact over short distances (paracrine signaling), or without contact over long distances and/or scales (endocrine signaling) [57]. Thus, in either reusing the existing cells or creating bio-inspired nano-machines able to copy their behavior, an analogous classification can be made. In bio inspired molecular communication, we distinguish between short range 
communication (referred to distances from nanometers to millimeters) and long range communication (referred to distances from millimeters to meters). In short range communication, molecular motors [52] and calcium signaling [54] are some of the proposed techniques in the literature. In long-range communication only pheromones usage has been proposed so far [1].

In this thesis novel bio-inspired techniques suitable for the long range will be proposed. The focus on bio-inspired approach has been chosen due to the features that it provides, which are envisaged to pave the way to long range nano-communications. Long range nano-communication is an unexplored research area with unique and powerful characteristics capable of major revolutions. The main objective of this thesis will be the study and the analysis of potential solutions to intercommunicate nano-machines at distances 10 orders of magnitude bigger than their dimensions (reaching devices in the macro domain, if necessary).

\subsection{Applications}

The range of applications in which nano devices are required is astonishingly wide. Five main areas can be identified:

i) Biomedical Applications: e.g., immune system support, bio-hybrid implants, drug delivery systems and applications within genetic engineering.

ii) Environmental Applications: e.g., biodegradation assistance, animal and biodiversity control, and air pollution control.

iii) Industrial and Consumer Goods Applications: e.g., development of new materials, manufacturing processes and quality control procedures, also for food and water quality control applications, and advanced fabrics and materials development.

iv) Military Applications: e.g., nuclear, biological and chemical (NBC) defences and nano-functionalized equipments.

v) Telecommunications, ICT and Future Internet: e.g., distributed execution and management of dynamic and intelligent services in unpredictable environments. 


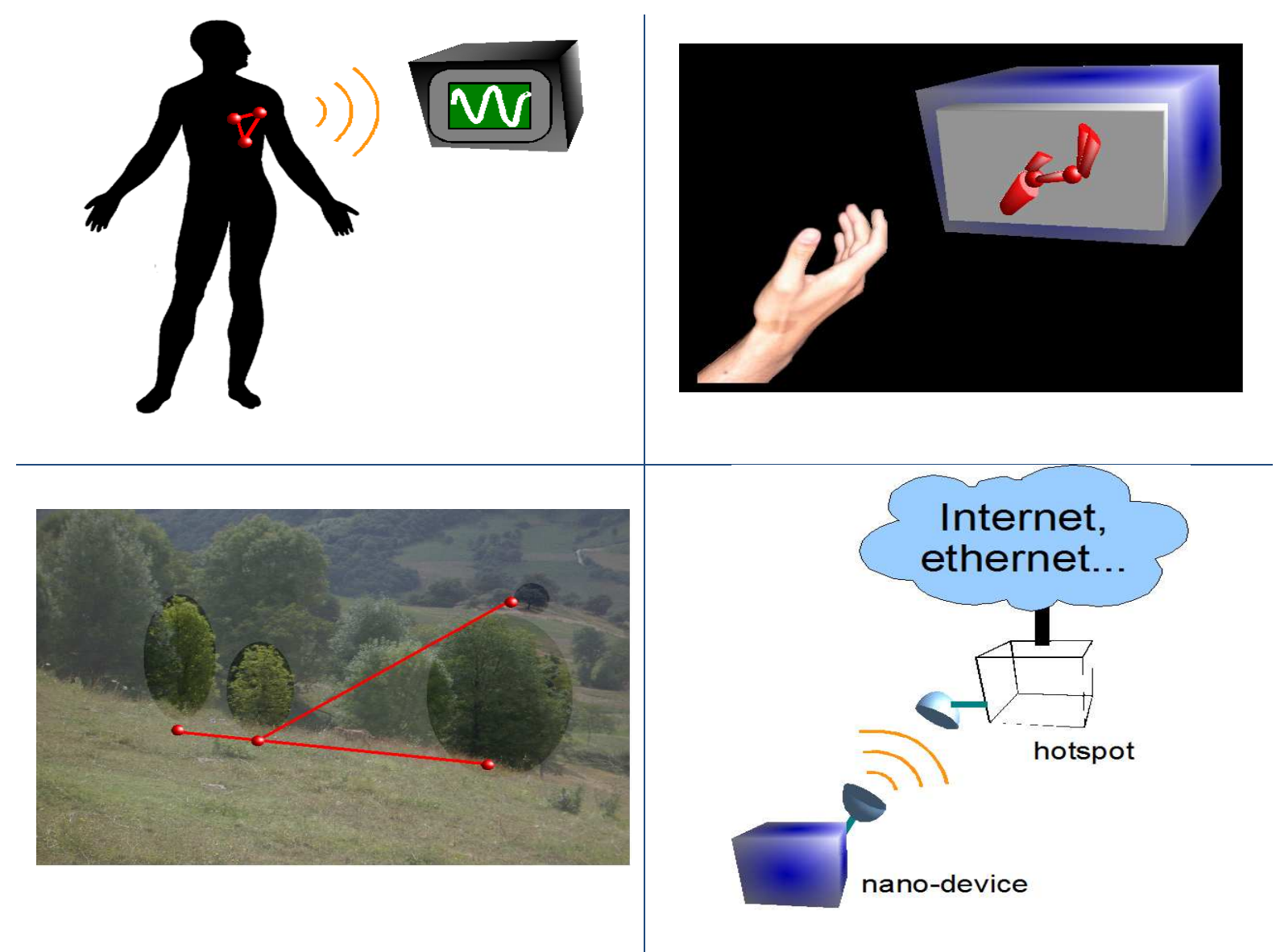

Fig. 1 Possible applications using long Range nanocommunication. Top-left: Medical APPS. (E.g., PATHWAY BETWEEN NANO-NETWORKS AND MACRO DEVICES). TOP-RIGHT: INDUSTRY APPS. (E.G., IMPROVEMENT OF HAPTIC INTERFACES IN VIDEOGAME INDUSTRY). BOTTOM-LEFT: ENVIRONMENTAL APPS. (E.g., ACCURATE SENSOR NETWORKS FOR TOXICITY, HUMIDITY...). BOtTOM-RIGHT: ICT APPS. (E.G., AD-HOC MOLECULAR NANO-DEVICES INTERCONNECTION AND MOleCUlar HOTSPOT ACCES).

Based on long range molecular communications, a wide variety of new applications can be designed. These applications would be impractical, or simply infeasible, if other classical communication techniques were used. The five main areas mentioned above can benefit from long-range molecular communication:

i) Biomedical Applications: long range communication will provide a pathway between intra-body nano-networks and macro devices (e.g., displays, oscilloscopes, cardiograms). In addition to this, the interconnection of different intra-body nano-networks or nano-systems can be achieved by taking advantage of long-range techniques (e.g., between heart monitoring nanonetwork and breathing system subnet). 
ii) Environmental Applications: retrieving different parameters (e.g., humidity in a forest, toxicity in a certain area) in environmental applications is a key feature that can be simplified by using nano sensors networks based on long-range molecular communications. In addition to this, the distribution of nano components able to sense environmental concentration of a substance would generate a more accurate output, as the sensing at the nano scale is able to detect chemical concentrations with a finer scale.

iii) Industrial and Consumer Goods Applications: the interconnection of tiny devices is a powerful feature that can be applied to industrial and consumer goods applications, increasing the value added of manufactured products. Amongst others, imperceptible nano-devices can be attached to usual products and provide new functionalities appreciated by potential consumers. A possible example is a network integrated by several sensors, which are embedded in a motorbike helmet and in the gloves of the motorbike rider, that are able to share information in order to improve the rider security. The embedded nano-sensors in the fabrics are potentially undetectable and could be mixed with textile fibers at much reduced cost. Another example is the design of haptic interfaces for the videogame industry, improving the player feelings and virtual movements in the designed game. Nano-sensors in videogame industry would be able to track player movements in a very accurate precision, and could even trigger nervous stimulus by transmitting an intra-body signal.

iv) Telecommunications, ICT and Future Internet: the evolution of the telecommunication world will be possible mainly through the miniaturization of communication devices from the current micro-scale to the nano-scale and through their efficient interconnection. In that scenario, long range molecular communication can be a means for nano-devices to reach distributed internet access points. Moreover, ad-hoc computers nano-networks will be able to be implemented with these techniques.

Regarding military field, a wide range of devices could be largely enhanced by the application of the techniques coming from the fields mentioned above. 


\subsection{DOCUMENT ORGANIZATION}

In this thesis, five options to implement the listed applications will be proposed. The suggested techniques, summarized in Fig. 2, are classified into two molecular communication schemes, either wired or wireless, on the basis of the signal propagation and the specific implementation target. Wireless communication options are referred to the communication techniques whose information transference does not require electrical conductors, or other physical link but a fluid medium (e.g., air, water, blood). Wired options are referred to the communication methods that require a physical link to transport the signal.

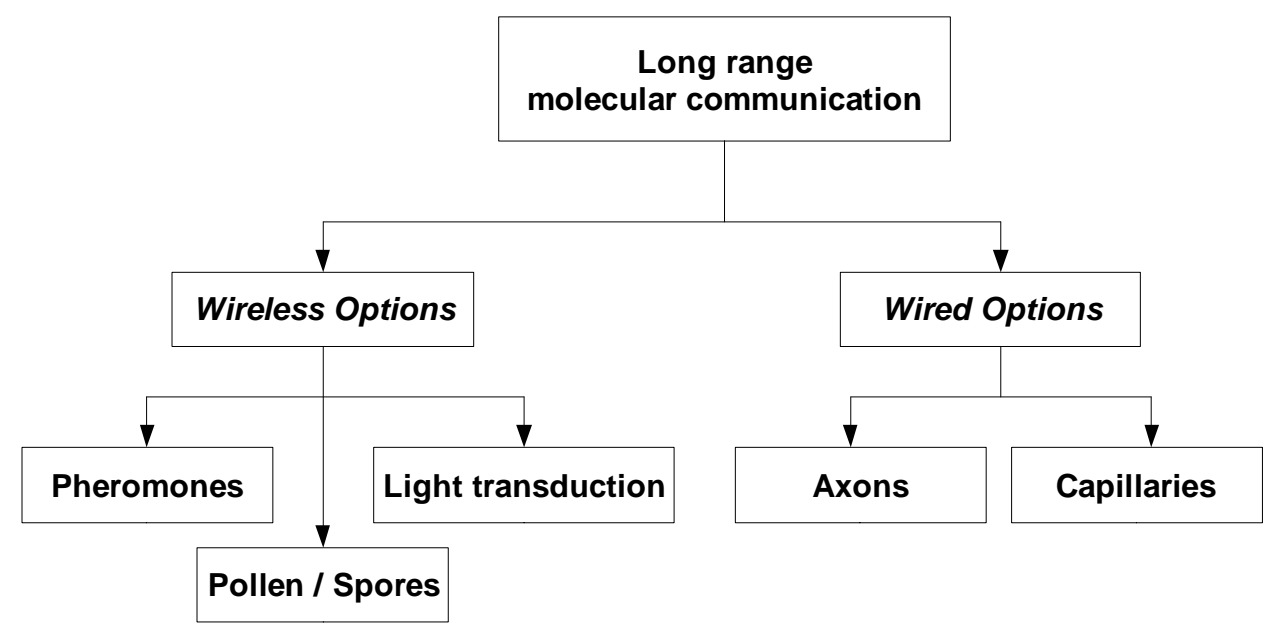

Fig. 2 Options for long RANGE MOLECULAR COMmUNicAtion

In this thesis, each of the proposed techniques will be analyzed according to the classical scheme division in communications (Fig. 3). Encoding and emission, propagation and reception and decoding will be the three sections in each of the suggested options. Some insights to existing models and the mathematical formulation of the critical parts will be also offered.

On chapter 2 the wireless molecular communication options are discussed, namely, the techniques based on pheromones, pollen and spores. In chapter 3 the wired communication options will be handled, including the techniques based on action potentials in axons, and flow circuit in capillaries. In each case, we discuss the usual communication steps, namely, encoding, emitting, propagation, receiving and decoding. In chapter 4 we qualitatively compare all the proposed schemes and discuss their advantages and drawbacks. In chapter 5 , the main conclusions of these long range molecular communication techniques are drawn up. 


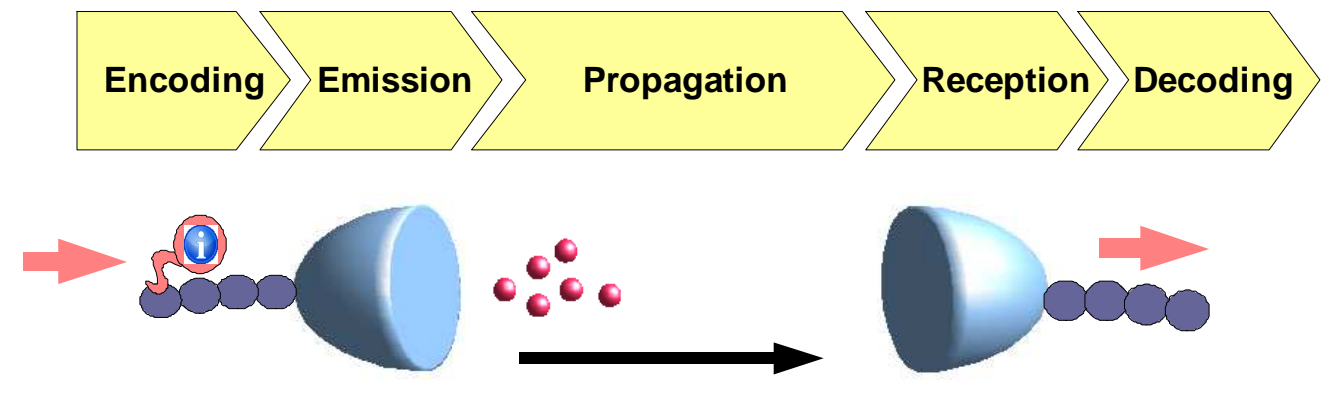

Fig. 3 Communication process stages that Will be used to anAlyze each of the PROPOSED TECHNIQUES. 


\section{WIRELESS OPTIONS}

Wireless molecular communication in the nano domain can be mainly realized through two different propagation techniques, namely, molecular diffusion and molecular transport in fluid medium. The optical wave propagation is also considered here as a bridging technique for the connection between the nano-world and the macro-world, and able to exploit wireless molecular communication.

Molecular diffusion stands for a spontaneous transport of molecules from a region of higher concentration to a region of lower concentration. This transport is the result of an underlying random molecular motion. Through the molecular diffusion process, the molecules released by a transmitting nano-machine can propagate through the medium until the destination. The transmission of pheromones is an important molecular diffusion based mechanism and it will be covered in subsection $A$.

Molecular transport in fluid medium can be applied when the communication molecules are grouped together into bigger entities, such as pollen and spores. Pollen and spores, due to their size and mass, cannot rely on molecular diffusion to propagate, but need to be transported by a flowing fluid medium (e.g., air or water). Pollen and spores transmission will be discussed in subsection $B$.

The optical wave propagation can be used to transmit a molecular communication signal to micro-electronic devices. In subsection $\mathrm{C}$ some insights about the possibilities of converting molecular information into optical signals and vice versa will be offered.

\subsection{PHEROMONES}

Pheromones are molecules of chemical compounds released by plants, insects and other animals that trigger specific behaviors among the receptor members of the same species. The use of pheromones for molecular communication in long range nanonetworks has been recently proposed in [1]. 


\subsubsection{ENCODING AND EMISSION}

Pheromones enable the members of a certain species to share information messages (e.g., pertaining alarm, food or mating) only with members of the same species. This mechanism is achieved by means of the establishment of communication channels involving the release, the propagation and the reception of pheromones. A wide range of different pheromones is present in nature. Each type of pheromone is characteristic of a particular species and it is uniquely bound to a precise message. This feature is denominated pheromone diversity. Hence, pheromone diversity allows different communication channels to be inherently free from interference among each other.

Pheromone diversity can be successfully applied to nano-machine communication following two different strategies. On the one hand, through the emission and reception of different pheromones it is possible to create different non-interfering channels (each channel will be using a different pheromone type). On the other hand, the use of different pheromones can be used in a single channel, thus increasing the information enclosed in the emitted pheromone molecules.

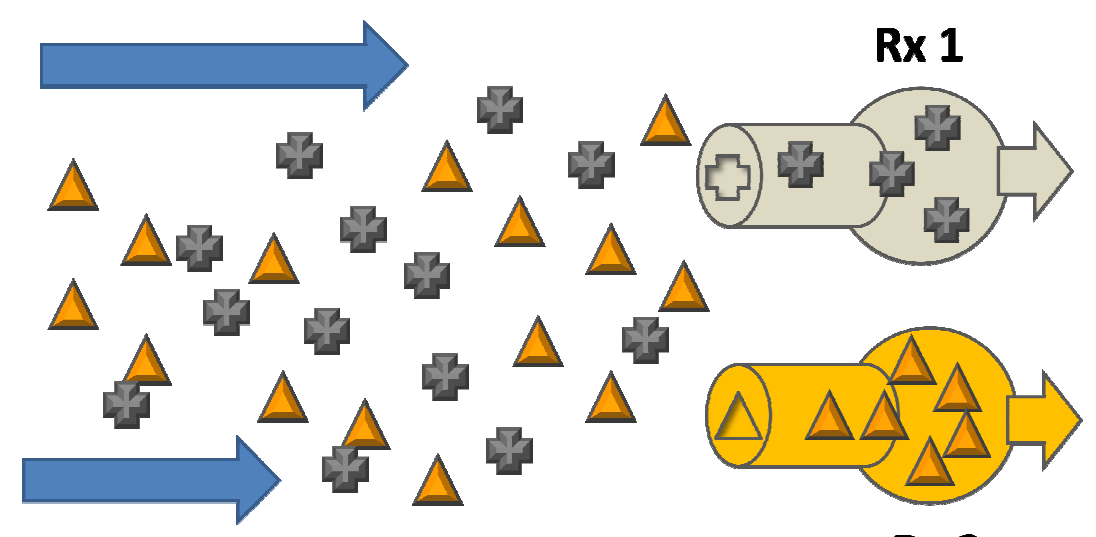

Rx 2

Fig. 4. USE OF PHEROMONE DIVERSITY TO CREATE NON INTERFERING CHANNELS.

When pheromone diversity is used to create different non-interfering channels among nano-machines, the emitting nano-machine takes the role of an insect sending a message using a certain type of pheromone. The message would only be understood by insects of the same species, as a result of possessing the specific pheromone receptor. Analogously, the receptor nano-machine must be equipped with the pheromone receptor able to detect that specific pheromone type. An illustration of this technique can be seen in Fig. 4, where crosses 
stand for one pheromone type and triangles represent a different pheromone type. The information can be encoded either in amplitude or in frequency, varying the concentration present in the medium of that specific pheromone. The use of pheromone diversity to create non-interfering channels can be thought as a Molecular Division Multiple Access (MDMA). In this scenario, the channels are separated by type of pheromones, in the same way that in FDMA the channels are separated in frequency and in TDMA the channels are separated in time slots.

Pheromone diversity can be used to increase the number of different molecules that can be emitted in a single channel to increase the alphabet size. In this case, the emitter nano-machine must be able to release different pheromones, and the receiver nano-machine must be equipped with as many different pheromone receptors as can be emitted by the transmitter nano-machine. Applying this technique the information throughput is boosted, since the information associated to each received molecule is increased. Indeed, if we consider the possibility of having 2 states for each pheromone, namely, released and not released, the amount of messages able to be transmitted is $2^{n}$, where $n$ is the number of different available pheromone types. As the receptor is equipped with a specific detector for each pheromone type, the signal components (the different pheromone types) are orthogonal and do not interfere with each other.

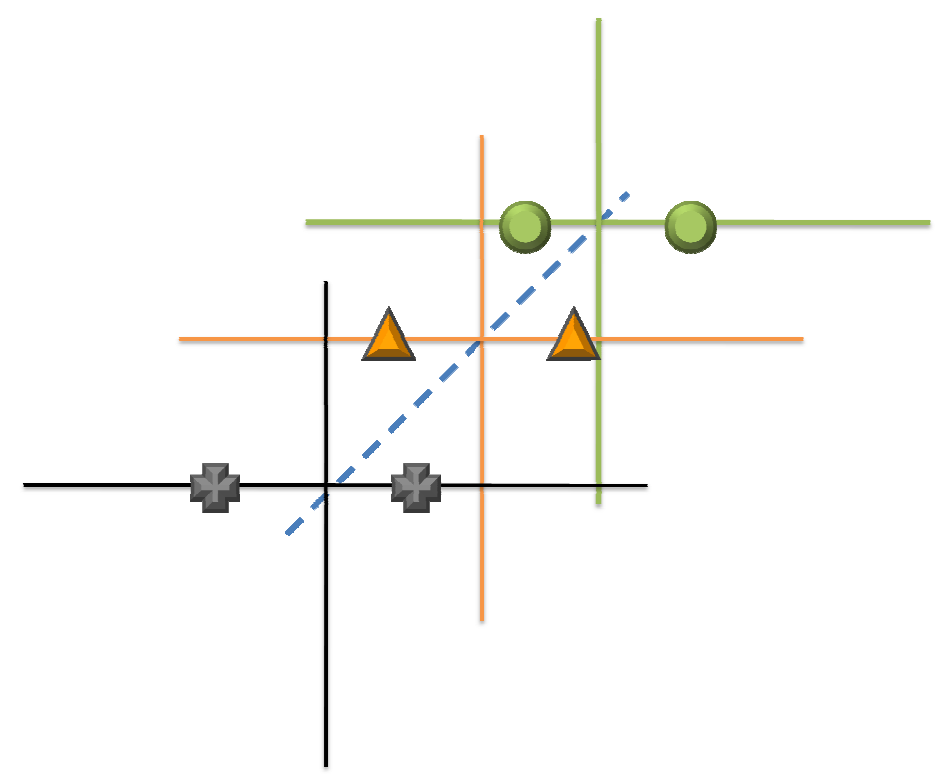

FIG. 5. USE OF PHEROMONE DIVERSITY TO CREATE INCREASE ALPHABET SYMBOLS. 
A combination of both schemes is also possible, optimizing the entire potential of pheromone diversity. For this, a specific subset of pheromones, $S_{1}$, is assigned to a communication channel between two nano-machines. Another subset, $S_{2}$, (where $S_{1}$ and $S_{2}$ are disjoint subsets) is assigned to another channel, possibly involving two different nano-machines. As a consequence, the transmitting nano-machines are able to encode information in more than one pheromone type, but without interfering with other nano-machine communications. More advanced protocols can manage the dynamic assignment of pheromone subsets to transmitting nano-machines, or decide to reserve a third subset to assign addresses to nano-machines and route the messages in a more efficient way.

The application of degrading enzymes is another technique that can be used in pheromone encoding. Enzymes are biomolecules (usually proteins) that increase the rates of chemical reactions; the degrading enzymes reduce the life of pheromone molecules, thus weakening the pheromone signal as a function of time. Altering the life time of pheromones will add more levels of freedom to the wireless molecular communication system, providing several features listed in the succeeding paragraphs.

First of all, priority mechanisms between different emitting nano-machines could be established with the usage of degrading enzymes. The priority mechanisms could be needed, for example, in the case that two different emitting nano-machines (each of them emitting with different pheromone types) try to reach the same nano-machine receptor. If the emitting nano-machines apply different enzymes to their emitted molecules, the receptor nano-machine receives different amounts (concentration) of the two types of pheromones depending whether the degrading enzyme acts faster or not. Then the receiver can assign a different priority depending on the concentration value of each pheromone type.

Another possible usage for degrading enzymes is the implementation of a mechanism similar to the Time To Live (TTL) used in conventional networks. In several already existing protocols (e.g., multimedia related), TTL feature is used to assure that a certain message will be delivered before the expiring of a defined amount of time. In pheromones case, applying the enzymes to emitted pheromone molecules would degrade the concentration intensity in a rate that depends on the particular applied enzyme. As the emitting nano-machine broadcasts the pheromone molecules in an isotropic fashion, the TTL feature could be useful to prevent known problems in this network topology. For example, TTL could be used to control the retransmission of the message by relay nano-machines, thus avoiding flooding in the network. 
TTL could also be used to limit the intermediate nano-machines that can be used from the emitter to the receptor (hops in multi-hop path).

Finally, it is also possible to use these enzymes for the evaluation of the transmitter-to-receiver nano-machine distance (or range). A further nano-machine with the enzyme applied to its emitted pheromones will reach the destination in a lower concentration than a closer emitting nano-machine. Knowing the applied enzyme to the emitted molecules and the molecular concentration at the emitter, it is possible to calculate the distance between emitter and receptor nano-machine.

\subsubsection{PROPAGATION}

Pheromones propagate in space by means of molecular diffusion. Molecular diffusion is a very complex mechanism deeply studied in entomology and botany, amongst other research fields. In the following, two simple models of pheromones diffusion in still air are exposed. These models are based on the Fick's laws of diffusion [61] and particularized for pheromone diffusion. Fick's first law of diffusion relates the diffusive flux to the molecular concentration, equation ( 1 ). Fick's second law of diffusion predicts how molecular concentration changes with time, as it can be seen in equation ( 2 ).

$$
\begin{aligned}
& J(r, t)=-D \nabla \mathrm{U}(\mathrm{r}, \mathrm{t}) \\
& \frac{\partial U(r, t)}{\partial t}=D^{2} \nabla \mathrm{U}(\mathrm{r}, \mathrm{t})
\end{aligned}
$$

where $J$ is the diffusion flux in $\frac{m o l}{\mathrm{~cm}^{2} \mathrm{~s}^{\prime}}, D$ is the diffusion coefficient in $\frac{\mathrm{cm}^{2}}{\mathrm{~s}}, U$ is the molecular concentration in $\frac{\mathrm{mol}}{\mathrm{cm}^{3}}$ and $r$ and $t$ are distance and time variables, respectively.

The models that will be discussed are differentiated by the emitting scheme. In the first one, exposed in subsection i), instantaneous emission is treated. In subsection ii) the continuous emission of pheromone molecules is considered. These models are analyzed assuming a single emitting nano-machine and a single receptor (peer-to-peer communication) and without 
analyzing possible interference with other molecular signals or remaining molecules in the medium. More advanced models on molecular diffusion are exposed on [62].

\subsubsection{INSTANTANEOUS EMISSION}

Instantaneous emission of pheromones is usually takes place when alarm situations occur in nature. In this kind of emission, the totality of a fixed amount of pheromones is released into the medium (e.g., air or water) in a minimized time, abruptly increasing the molecular concentration around the emitting nano-machine. Then, due to molecular diffusion, these molecules will travel through the medium dispersing themselves randomly.

The sudden release of a fixed amount of particles (instantaneous emission) is the most suitable modeling when pheromone diversity is used to increase the molecules that can be emitted by a single nano-machine. In this case, we are not interested in variation in molecular concentration (amplitude or frequency modulation) but in the type of emitted pheromones. Thus, there is no need to continuously fill the medium with molecules and the puff emission is the most suitable scenario.

For a still air and no molecular interference scenario, the pheromone density $U(r, t)$, in $\mathrm{mol} / \mathrm{cm}^{3}$, can be obtained from Robert's equation [7]:

$$
U(r, t)=\frac{2 Q}{(4 \pi D t)^{\frac{3}{2}}} e^{\frac{-r^{2}}{4 D t}}
$$

where $r$ is the distance to the emitting source in $\mathrm{cm}, t$ is the time from emission start, $Q$ is the amount of released molecules and $D$ is the diffusion constant in $\mathrm{cm}^{2} / \mathrm{s}$, which depends on the propagation medium.

In Fig. 4, equation ( 3 ) is used to illustrate the delay and maximum concentration detection in distances ranging from $2 \mathrm{~cm}$ to $2.6 \mathrm{~cm}$ from emitter. With the parameters used $(Q=100,000$ $\mathrm{mol}$ and $D=0.43 \mathrm{~cm}^{2} / \mathrm{s}$ ) and assuming a receptor molecular threshold $C=1,000 \mathrm{~mol}$, the maximum radius is approximately $2.44 \mathrm{~cm}$. Indeed, at $2.4 \mathrm{~cm}$ from the emitter the maximum concentration received is $1,065 \mathrm{~mol} / \mathrm{cm}^{3}$, and at 2.6 it is $837 \mathrm{~mol} / \mathrm{cm}^{3}$. Longer radius can be 
reached by increasing the $\mathrm{Q} / \mathrm{C}$ relation (molecules released / threshold molecules). As an example, the alarm pheromone of the ant Acanthomyops claviger has an effective radius of 10 $\mathrm{cm}$ and signals take 2 minutes to reach this distance and 8 minutes to fade out [42]. Regarding the delay, it is necessary 1.7 seconds to reach $2.4 \mathrm{~cm}$.

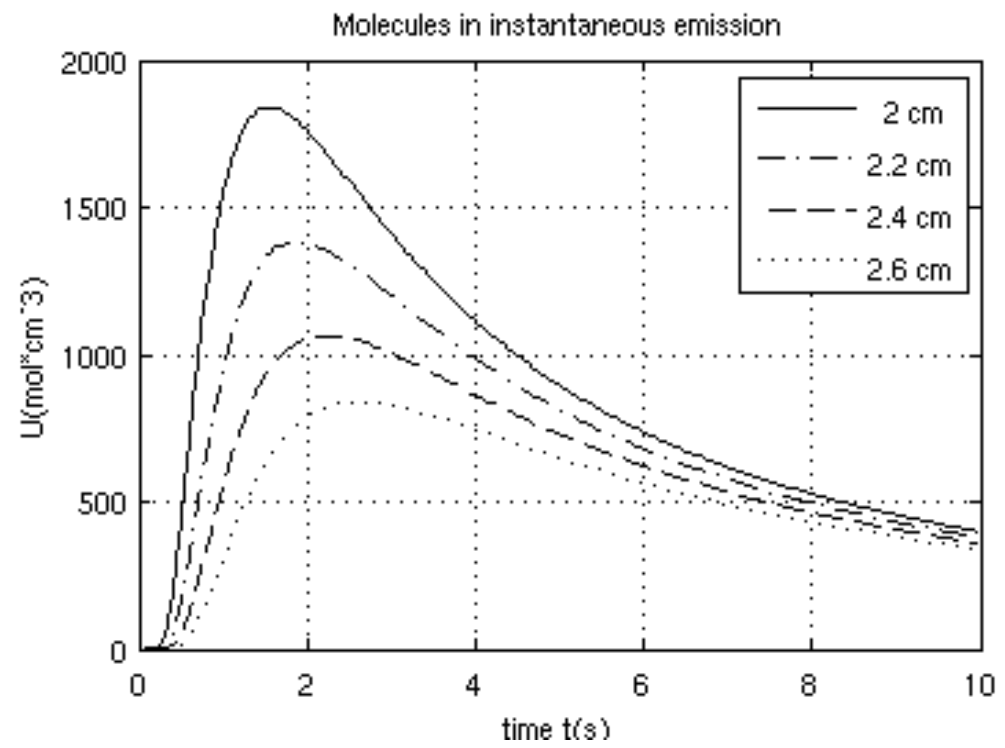

Fig. 6. Pheromonal density, according to diffusion equation ( 3 ), as a function of TIME FOR DIFFERENT DISTANCES FROM EMITTER. THE DIFFUSION COEFFICIENT IS D $=0.43 \mathrm{~cm}^{2} / \mathrm{s}$, THE AMOUNT OF RELEASED MOLECULES IS $Q=100,000$ AND THE THRESHOLD OF THE MOLECULES IN RECEPTION IS $\mathrm{C}=1000$ MOLECULES.

\subsubsection{CONTINUOUS EMISSION}

When pheromone diversity is used to deploy a molecular division multiple access (MDMA), the information is encoded in amplitude or frequency variation of pheromone molecular concentration. In amplitude or frequency molecular modulation, the continuous emission is needed. If the emission rate of molecules is constant $Q(\tau)=Q$, for continuous emission in still air the pheromones propagation is [7]:

$$
U(r, t)=\frac{Q}{2 D \pi r} \operatorname{erfc}\left(\frac{r}{\sqrt{4 D t}}\right)
$$

where erfc is the complementary error function: 


$$
\operatorname{erfc}(x)=\frac{2}{\sqrt{\pi}} \int_{x}^{\infty} e^{-v^{2}} d v
$$

With the same $Q / C$ relation $(Q=100,000$ and $C=1,000)$, we can use equation ( 4 ) to visualize the transmission delay in continuous emission scheme. As it can be seen in Fig. 5, in this case greater distances can be achieved, but the time needed to reach the receiver is nonlinearly increased. As an example, to reach the threshold $C$ at $1 \mathrm{~cm}$ it is needed 1 second, whereas to reach the same concentration at $10 \mathrm{~cm}$ it is needed around 95 seconds.

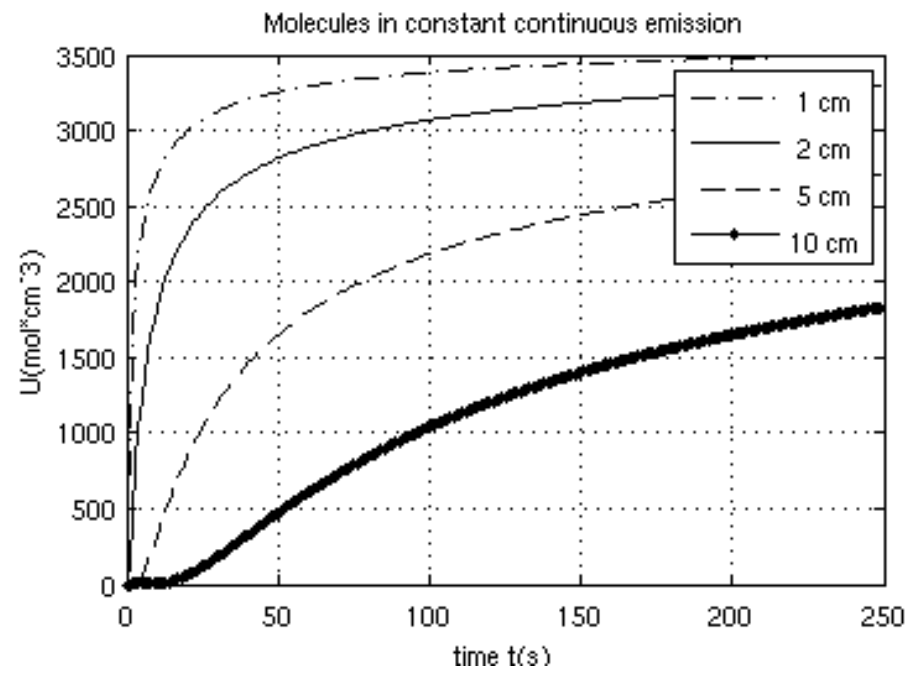

Fig. 7. Pheromone molecules density trough time for different distances from EMITTER, ACCORDING TO DIFFUSION EQUATION ( 4 ). THE DIFFUSION COEFFICIENT IS CHOSEN $D=0.43$ $\mathrm{CM}^{2} / \mathrm{s}$, THE AMOUNT OF RELEASED MOLECULES $Q=100,000$ AND THE THRESHOLD OF THE MOLECULES IN RECEPTION IS C $=1000$ MOLECULES.

So far, the presence of wind has not been considered. On the one hand, it can boost coverage or transmission speed, carrying pheromone molecules further on its flow. But on the other hand, wind implies turbulences that can produce distortion and molecule dispersion, affecting the signals. Moreover, subordinating the transmission to such external and varying factor is impractical, unless it is fixed or very regular (e.g., conduct the flux and generate air movement if needed). 


\subsubsection{DeCoding And RECEPTION}

As a result of the high molecular diversity of pheromones, natural receptor mechanisms are highly sensitive and selective. As shown in Fig. 6, pheromone antenna has three molecular filters [43]. The first one is the cuticle and has some gates, known as pore tubules, which allow pheromones to get inside the antenna core, namely, the sensillar lymph. After passing the cuticle, pheromones are encapsulated by odorant binding proteins (OBP). The OBP-ligand complex reaches the dendrite olfactory receptors, triggering an electrical signal. Hence, this antenna is able to convert from a pheromone to an electrical signal. Nano-machines equipped with these antennas will be able to understand the received electrical signal and react accordingly.

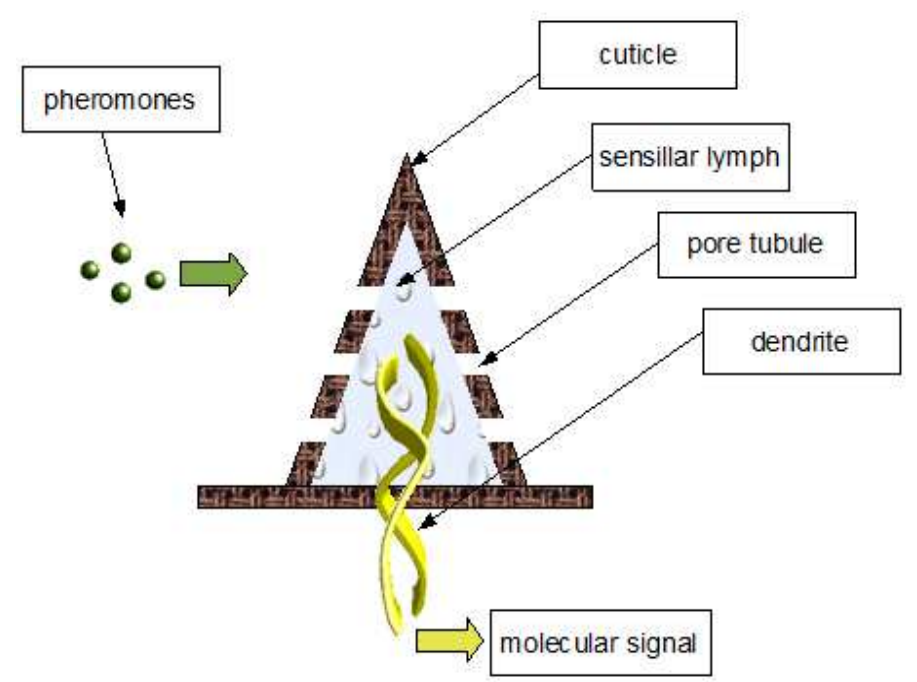

Fig. 8 ANTENNA StRUCtURE For PHEROMONE RECEPTION, ACCORDING to [43].

\subsubsection{MODEL}

The dispersion of pheromone molecules through the space has been largely studied, mostly in entomology field. Almost all of the models include in its formulation the medium turbulence as an inherent factor. This variable it might not be necessary in short range coverage $(1 \mathrm{~mm}$ to $10 \mathrm{~cm}$ ), but it is essential for larger distances due to the attenuation and transmission speed of such method of communication. As an example, in Fig. 7 the attenuation of particles as a result of its diffusion is depicted as a function of distance and frequency: 


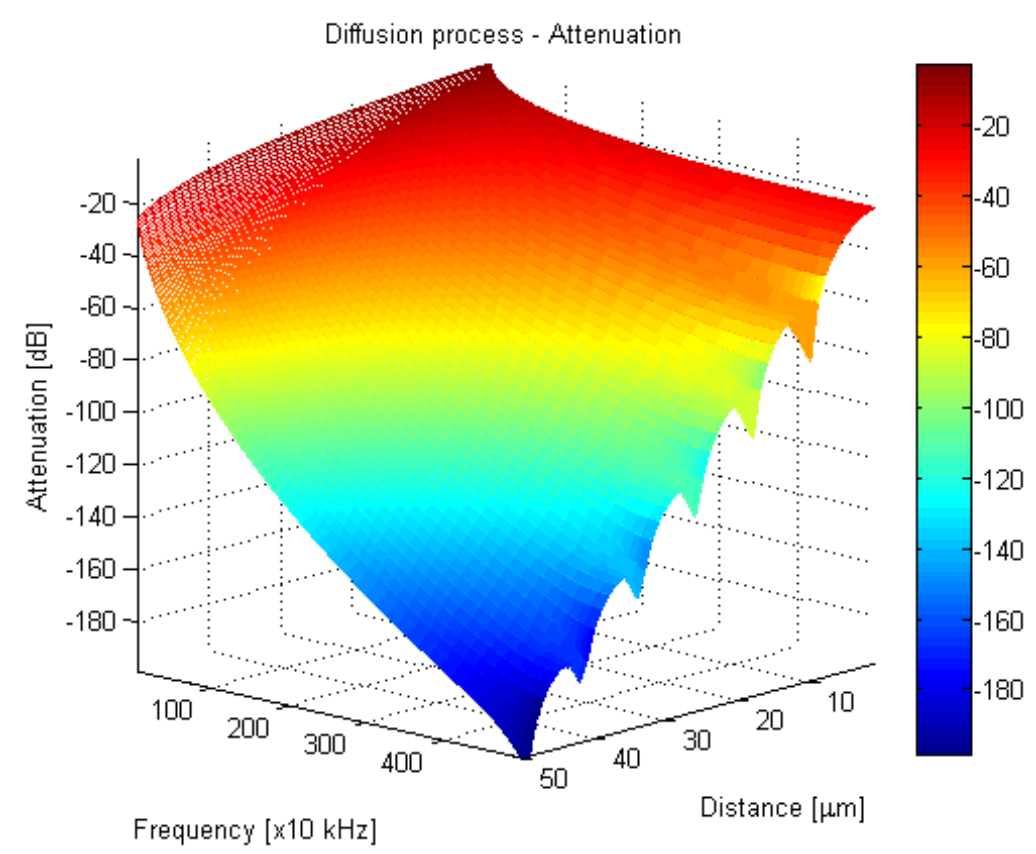

Fig. 9. Attenuation in a molecular diffusion process as a fUnction of distance AND FREQUENCY, ACCORDING TO [62].

As it can be seen, even for a continuously emitting source $(\mathrm{f}=\mathrm{OHz})$, at 50 micrometers the attenuation is $-30 \mathrm{~dB}$. If we need to include frequency in our modulation scheme, the overall attenuation decreases up to $180 \mathrm{~dB}$ for $5 \mathrm{MHz}$. The graph is realized in [62] after the demonstration of the validity of the Diffusion Green's Function. Firstly, the concentration is decomposed using Fourier orthonormal bases:

$$
c(\bar{x}, t)=\int_{K} A_{\bar{k}}(t) e^{-j \bar{k} \bar{x}} d \bar{k}
$$

where $\bar{k}$ is the vector containing the spatial frequencies, one for every dimension of $\mathrm{S}$, spanning the spatial frequency domain K. Substituting $c_{k}(t)$ into the Fick's Second Law Partial Differential Equation, a solution for any spatial frequency component is found.

$$
\begin{gathered}
A_{\bar{k}}(t)=A_{\bar{k}}(0) e^{-D\|\bar{k}\|^{2} t} \int_{K} A_{\bar{k}}(0) e^{-D\|\bar{k}\|^{2} t} e^{-j \bar{k} \bar{x}} d \bar{k} \\
A_{\bar{k}}(0)=\frac{1}{(2 \pi)^{n_{d i m}}} \int_{S} c(\bar{x}, 0) e^{j \bar{k} \bar{x}} d \bar{x}
\end{gathered}
$$


Although the development of the previous model can give expressions for short range molecular communication (e.g., ion signaling), for long range molecular communication the presence of medium turbulence (e.g., wind) cannot be neglected. Among all the models, the Sutton model and the Gaussian Plume model are the most common models applied to pheromone diffusion [22]. As focused on atmospheric surface layer, none of the mentioned models consider other forces like Coriolis, geostrophic winds and molecular viscosity.

\subsubsection{SUTTON MODEL:}

Sutton developed this model in 1953, being first applied to pheromones by Bossert and Wilson in 1963 . The model describes the pheromone concentration $C$ for every point in the tridimensional space xyz.

$$
C(x, y, z)=\frac{2 Q}{\pi C_{y} C_{z} U x^{2-n}} e^{\left[-x^{n-2}\left(\frac{y^{2}}{C_{y}^{2}}+\frac{z^{2}}{C_{z}^{2}}\right)\right]}
$$

where $Q$ is the release rate, $U$ is the mean wind speed (aligned with $x$ axis), $C_{y}$ and $C_{z}$ are the respective horizontal and vertical diffusion coefficients, and $n$ is a parameter $(0<n<1)$ dependent on the vertical profile of wind velocity.

The dispersion coefficients are functions of the atmospheric turbulence, terrain roughness, and vertical windspeed profile. In [71], it is suggested to use $\left\{C_{y}=0.4 \mathrm{~cm}^{1 / 8}, C_{z}=0.2 \mathrm{~cm} 1 / 8, n=\right.$ 0.25 for light winds and neutral atmospheric conditions.

For an elevated source, Sutton suggests the following equation:

$$
C(x, y, z, h)=\frac{Q \cdot e^{\frac{-y^{2}}{C_{y}^{2} x^{2-n}}}}{\pi C_{y} C_{z} U x^{2-n}}\left[e^{\frac{-(z-h)^{2}}{C_{z}^{2} x^{2-n}}}+e^{\frac{-(z+h)^{2}}{C_{z}^{2} x^{2-n}}}\right]
$$

where $h$ is the height of the source above ground. 


\subsubsection{Gaussian Plume model:}

In the Gaussian plume model all variables are defined as in the Sutton model, except that the horizontal and vertical dispersion coefficients $\left(\sigma_{y}, \sigma_{z}\right)$ are functions of the downwind distance $x$, and a new parameter $\alpha$ is defined as a constant $(0<\alpha<1)$ that depends upon the degree of absorption of material to the ground.

$$
C(x, y, z, h)=\frac{Q \cdot e^{\frac{-y^{2}}{2 \sigma_{y}^{2}}}}{2 \pi \sigma_{y} \sigma_{z} U}\left[e^{\frac{-(z-h)^{2}}{2 \sigma_{y}^{2}}}+\alpha \cdot e^{\frac{-(z+h)^{2}}{2 \sigma_{z}^{2}}}\right]
$$

As it can be seen in Fig. 8 and Fig. 9, this model describes reasonably well the deposition of particles taking into account turbulence in the medium. Moreover, for more advanced models, section 2.2.4 offers some developments that can be applied to pheromonal diffusion.

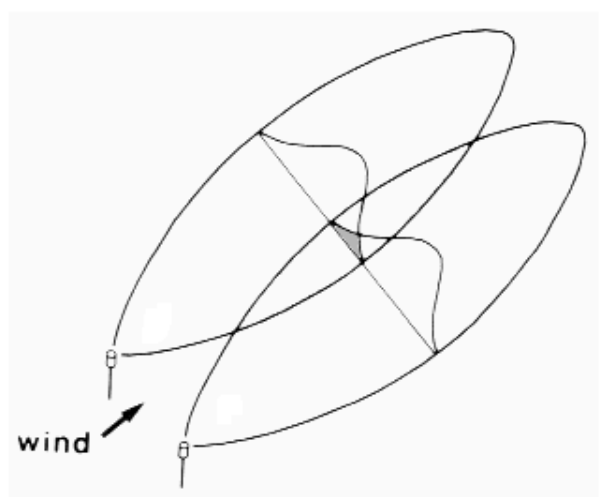

Fig. 10 GaUsSian PLUME, ACCORDING to [11]

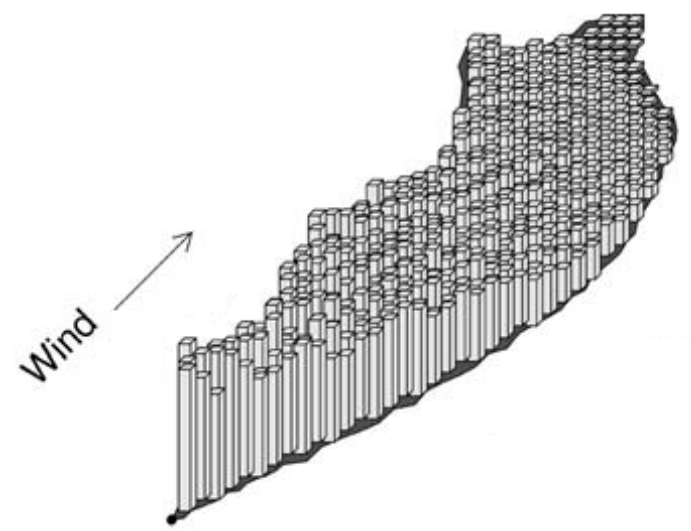

Fig. 11 ACtive SPACE IN WHICH PHEROMONE CONCENTRATION IS ABOVE A BEHAVIORAL THRESHOLD, INDICATIONG THE PROBABILITY OF AN INSECT REACHING THE SOURCE [10] 


\subsection{POLLEN AND SPORES}

Pollen and spores are reproductive structures adapted for dispersal (pollen in plants and spores in fungi). Although bigger than pheromones (smallest pollen grain, from Myosotis, is $6 \mu \mathrm{m}$ in diameter and spore from Aspergillus is $2 \mu \mathrm{m}$ to $6 \mu \mathrm{m}$ ), they can offer some advantages presented in the following sections.

\subsubsection{ENCODING AND EMISSION}

Like pheromones, pollen and spores can offer the particle diversity propriety; there exist different packets (e.g., types of particles) that can only be understood by certain receivers. This feature can aid the encoding protocol (enlarging the possible alphabet) or improve the routing (e.g., identifying each channel by different particles usage).

However, in the pollen case the possibility of encoding DNA in each molecule is a great feature that can widely expand the symbols to transmit and improve the channel throughput. As both pollen and spores are vegetal reproduction methods, they enclose the parent DNA in its structure. DNA chain decoding can be done in the receptor node in order to obtain the information embedded in it. Pollen manipulation is a well studied technique, widely covered in the literature such as in [79] and [19].

\subsubsection{PROPAGATION}

As far as the authors know, there is not any theoretical mathematical model of pollen or spore propagation. Fick's laws and diffusion models studied in pheromones cannot be applied in the case of pollen and spores. In pollen and spores case, the diffused particles have bigger dimensions, and some previously obviated factors have determinant effects in this new scenario. Although pollen and spores dispersion has been largely studied in botanical and mycological fields, only empirical research can be found. The lack of a solid theoretical model for these particles dispersion can be attained to the complexity and the big number of factors affecting the deposition processes.

The propagation of pollen and spores has been studied based on empirical measures and its fitting to approximate expressions. The first equation was exposed in [30], where the amount $p$ of pollen deposition was calculated as a function of distance as shown in ( 12 ): 


$$
p(r)=\frac{Q^{\prime} e^{-b r}}{r}
$$

where $Q^{\prime}$ is the amount of pollen released to the medium, $r$ is the distance from the source and $b$ is a constant indicating the proportion of pollen per distance unit $(b \geq 0)$.

To include the effects of turbulence, the previous equation is modified in [4]:

$$
p(r)=\frac{Q^{\prime} e^{-b r(1-m)}}{r^{1+m}}
$$

where $m$ is a turbulence parameter with $m=0.62$ for minimum turbulence and $m=0.88$ for maximum turbulence [4].

An improved version of equation ( 13 ) is exposed in [28], powering the exponential to a Gaussian function $\alpha+\beta \times \Phi(\theta: w, s)$. This modification provides a more accurate match to empirical measures.

$$
p(r, \theta)=Q^{\prime}\left(\frac{e^{-b \times r^{d}}}{r^{c}}\right)^{\alpha+\beta \times \Phi(\theta: w, s)}
$$

where $d$ and $c$ are a generalization of the parameter $m, w$ is the mean direction of the wind and $s$ its standard deviation. $\alpha$ and $\beta$ are parameters of the normal distribution function.

Pollen release of Lolium species has been empirically measured in [28], and modeled using equation ( 14 ). Anthesis (the period during which a flower is fully open and functional) is divided in four stages (early, mid1, mid2, late). In Fig. 10 pollen distribution in mid1 is represented with the following parameters: $w=121.01^{\circ}, s=62.27 ㅇ, Q^{\prime}=399.60 \mathrm{~mol}, b=0.2800$ $m^{-0.819}, c=0.819, d=0.607, \alpha=1, \beta=-35.860$. 


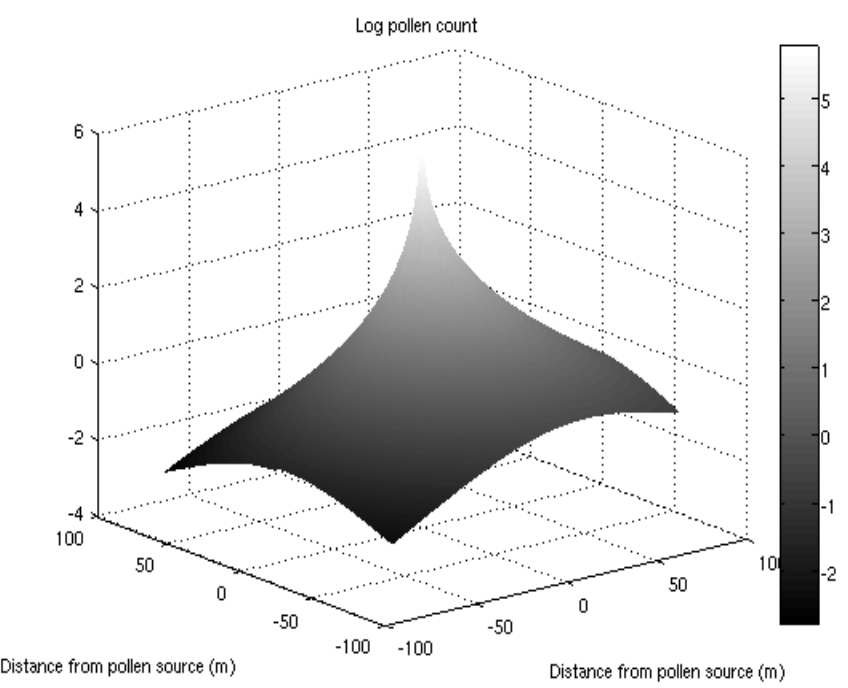

Fig. 12. Pollen Distribution in first half of Mid ANTHeSis (LOG COUNT).

An advantage of pollen propagation is that both pollen and spores are more robust to chemical reactions than pheromones. External chemical compounds in the medium will not modify the structure of pollen particles, whereas could provoke a chemical reaction in pheromones molecules.

\subsubsection{DECODING AND RECEPTION}

The main advantage of pollen or spores utilization is the possibility of DNA encoding. The most used technique to extract the DNA strands is hybridization, and it could be used in our receptor to decode the information enclosed in the DNA sequence. In hybridization process, the received molecules are heated to separate the complementary strands (thus splitting the DNA sequence). The breakage is possible because the hydrogen bonds that join the DNA base strands become thermodynamically unfavorable. After the complementary strand breakage, the nucleotides (structural units of RNA and DNA) will bind to their complement. If our receiver has different single stranded binding candidates, the received message will consist of successful DNA double strands. 


\subsubsection{MODEL}

In the transmission using pollen or spores, the key factor is the wind dispersal model. The presence of a turbulent medium (e.g., wind, fluid medium) is fundamental for the success of this communication technique.

The basis of several mechanical models of airborne particles is the ballistic equation. Using this equation, the average distance $d$ traveled by a released particle is [55]:

$$
d=\frac{\mathrm{h} \cdot \mathrm{w}}{\mathrm{v}_{\mathrm{t}}}
$$

where $h$ is the height where the particle is released, $w$ is the horizontal wind velocity and $v_{t}$ is the terminal velocity of the particle.

Although equation ( 15 ) is a good starting point for particles with a terminal velocity higher than $1 \mathrm{~m} / \mathrm{s}$, in pollen and spores case the upward movement caused by vertical turbulence is more important than falling caused by gravity. For this, the ballistic equation is not enough to predict the dispersal distance [38].

As [38] states, pollen and spores models can be grouped following two main approaches: Eulerian and Lagrangian. The Eulerian modeling approach focuses on the density of the particle pattern and, thus, explains the dispersal of an individual particle by modeling the probability of finding it in a given area. The Lagrangian approach models the movement of the particle itself, typically by simulating trajectories for dispersing particles.

From the communication perspective, Lagrangian models offer a better accuracy in transmission parameters. Although the receptor would be able to sense the concentration of pollen/spores, the evolution of the seed will describe as well the evolution of the transmission item if the packet is assigned to a single seed (pollen/spore). Parameters like attenuation, distortion or scattering will be embedded in the Lagrangian models, allowing further research to clearly identifying each factor contribution. 
In most of the studied models, the presence of wind is a key factor on the equations. In [36], the displacement of individual pollen grains is calculated by solving horizontal and vertical air velocity components. The joint stochastic equations used are:

$$
\begin{gathered}
\mathrm{d} u=a_{u} \mathrm{~d} t+b_{u} \mathrm{~d} \xi_{u} \quad \mathrm{~d} x=u \mathrm{~d} t \\
\mathrm{~d} w=a_{w} \mathrm{~d} t+b_{w} \mathrm{~d} \xi_{w} \quad \mathrm{~d} z=\left(w-V_{s}\right) \mathrm{d} t
\end{gathered}
$$

where $u$ and $w$ are the horizontal and vertical air velocity, $a_{u}, b_{u}, a_{w}$, and $b_{w}$ are the Langevin coefficients [77] and $\mathrm{d} \xi_{w}$ and $\mathrm{d} \xi_{u}$ are random numbers drawn from Gaussian distributions with mean zero and variance $\mathrm{dt}$.

One of the outputs of this model in [36] is the pollen concentration using the following equation:

$$
C(i, j)=V_{s} \cdot \frac{1}{N_{P}} \sum_{n=1}^{N_{P}} \frac{T_{n}(i, j)}{V(i, j)}
$$

where $V_{s}$ is the volume of the source, $N_{P}$ is the number of trajectories, and $T_{n}$ is the residence time of the particle $n$ in the grid division of volume $V(i, j)$.

Other model proposed in [69], strats from the following general equation for dispersal trajectory $\left(\mathrm{x}_{1}=\mathrm{x}, \mathrm{x}_{2}=\mathrm{y}, \mathrm{x}_{3}=\mathrm{z}\right)$ :

$$
x_{i}(t+\Delta t)=x_{i}(t)+\int_{t}^{t+\Delta t}\left(u_{i}-v_{t} \delta_{i 3}\right) d t \quad i=1,2,3
$$

where $u_{i}$ is the instantaneous wind velocity in direction $x_{i}, \Delta t$ is the discrete simulation time interval, $v_{t}$ is the seed terminal velocity, and $\delta_{i 3}$ is 1 for $i=3$ and 0 otherwise. 
From equation ( 19 ) several degrees of model complexity can be developed, such as stochastic models or Markov chain models. The simplest model is the deterministic, which for distances $<15 \mathrm{~m}$ is a good approximation. The deterministic model assumes that the dispersal distance depends only on the height the pollen/spore is released and the wind it experiences during this time. The mean horizontal wind velocity $U$ is calculated in the following form:

$$
U(z)=\frac{u_{*}}{k} \ln \left(\frac{z-d}{z_{0}}\right)
$$

where $u_{*}$ is the friction velocity, $z$ is the height from the ground surface, $d$ is the zero-plane displacement height, $z_{0}$ is the momentum roughness length, and $k=0.4$ is the Von Karman constant.

The dispersal distance $D$ can be calculated, then, using the following equation:

$$
D=\frac{u_{*}}{k \cdot v_{t}}\left[\left(H_{0}-d\right) \ln \left(\frac{H_{0}-d}{e \cdot z_{0}}\right)+z_{0}\right]
$$




\subsection{LIGHT TRANSDUCTION}

For light transduction we refer to the conversion between short range molecular information (e.g., calcium signaling) and optical signals. Future coexistence between nano and micro networks is unavoidable and their interconnection is an open challenging issue. Proposed techniques in this section will enable the interfaces of nano and micro devices, using optical signal as common carrier. The usage of optical conversion techniques will also enable gateways between several isolated short-range nano-networks. In this case, molecular signals are converted to optical waves, which are propagated in optical domain and converted back from optical to molecular information. The architecture's scheme is shown in Fig. 11.

Light transduction offers exciting features. For instance, it has an extremely high velocity compared to molecular signals. In addition to this, modeling of optical signals is extensively studied [6],[29] (e.g., in terms of attenuation, noise sources, bandwidth, etc). Inorganic components such as amplifiers or filers could be used in this pathway if needed.

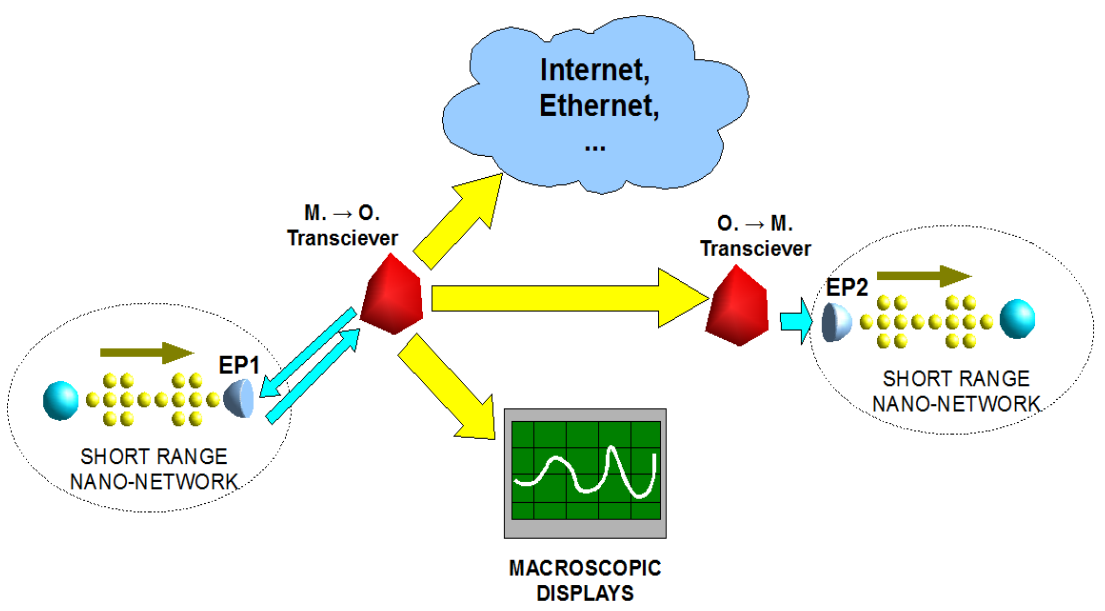

Fig. 13. Light tRANSDUCtion technique SChEME.

\subsubsection{ENCODING AND EMISSION}

Two novel solutions are proposed to allow the conversion from the molecular to the optical domain, namely fluorescent proteins and Molecular Organic Light Emitting Diode (MOLED's).

\subsubsection{FLUORESCENT PROTEINS}

Fluorescent proteins are biological molecules composed of aminoacids that fluoresce at certain wavelength when exposed to different wavelengths. These molecules have been 
developed since the cloning of the green fluorescent protein (GFP) from the jellyfish Aequoria victoria. Nowadays we can use from blue to red wavelengths (Fig. 12), each of them with different quantum yields (efficiency) and other parameters, like $\mathrm{pH}$ dependence. For green, yellow and red proteins the quantum yield (that measures the photonic efficiency) is above $60 \%$, in cyan is $40 \%$ and blue is as low as $25 \%$ [60].

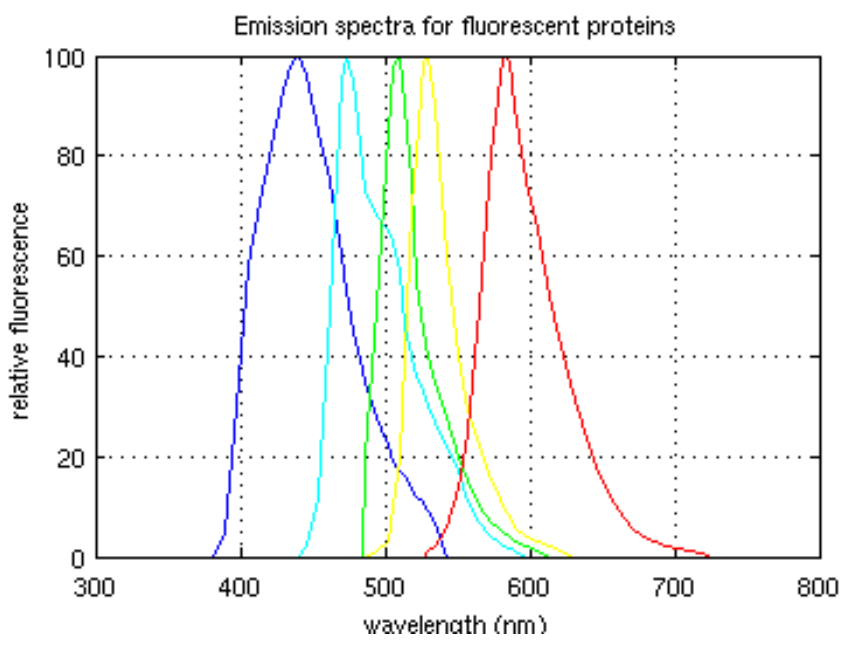

Fig. 14. Spectral emission of blue, Cyan, green, yellow and Red fluorescent proteins (LEFT to Right CURVES IN THE FigURE), ACCORDING TO [60].

Sensing the presence of these proteins (exciting them in the right wavelength), is a method currently in use for labeling molecules in biomedical research. Regarding telecommunications, it could also be used as an interface between a short-range nano-network and a long-range optical gateway. As it is shown in Fig. 11, the molecular to optical component $M \rightarrow O$ transceiver would be required to sense the presence at the endpoint node EP1 of the molecular signal. The $M \rightarrow O$ transceiver would emit a laser signal of approximately $10 \mathrm{~mW}$, retrieving the molecular information in the endpoint node of the short-range molecular network. Although the size of the transceiver is currently in macro domain, research on both nano-lasers [2], [46] and fluorescent proteins is currently in progress. This research is envisaged to optimize the fluorescent proteins properties (e.g., quantum yield, power requirement) to fulfill communications expectations. For example, in [14] a new fluorescent protein with novel characteristics is designed and tested. 


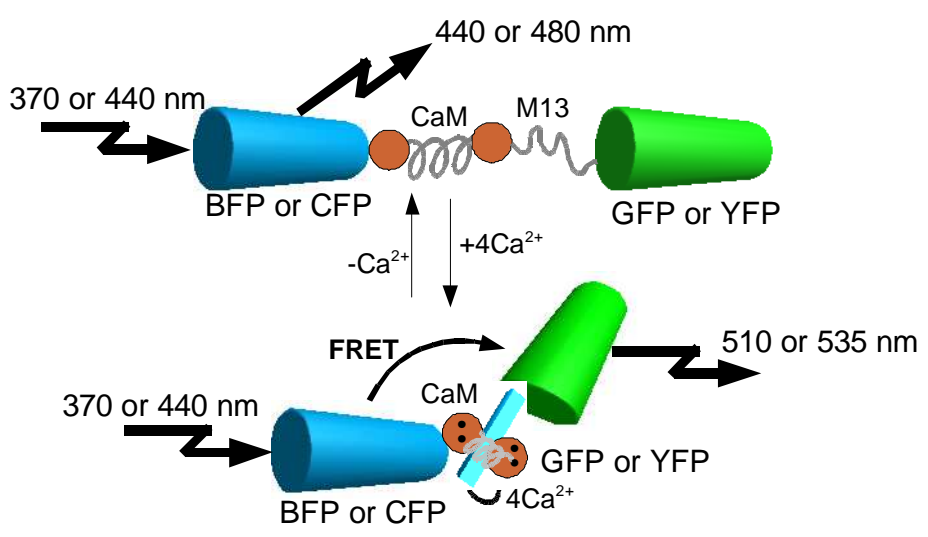

Fig. 15. CAlCiUm ions detection using fluorescent proteins, according to [51]

Of a particular interest is [51], where a fluorescent protein is described to detect presence of calcium ions. As calcium signaling is one of proposed methods for short-range communication, it is a clear interface to convert information from the $\mathrm{Ca}^{2+}$ to optical signaling. The sensing system is shown in Fig. 13. When blue or cyan fluorescent proteins (BFP/CFP) are excited, a light emission will be produced at 440nm (BFP) or 480nm (CFP) if there are no calcium ions and at $510 \mathrm{~nm}$ (GFP) or $535 \mathrm{~nm}$ (YFP) if there is Ca2+. GFP and YFP stand for green and yellow fluorescent protein, respectively. FRET stands for Förster resonance energy transfer, the mechanism of energy transfer between donor and acceptor protein.

\subsubsection{MOLED'S}

Molecular Organic Light Emitting Diodes or MOLED's are semi-conductor structures in the nano-scale that can be used to convert molecular signals into optical information. One of the main consequences of their tiny dimensions is that they have properties between bulk semiconductors and discrete molecules [9].

Although MOLEDs are designed downscaling organic LED's and not following a bio-inspired approach, methods for conjugating them to biomolecules are described in [27] using antibodies or peptides. Techniques to enclose these components in a biological shield and attach them into the target molecules have been already tested in medical research. This procedure offers some advantages to fluorescent proteins. Among others, MOLEDs have higher resistance to biological agents, much longer life and higher efficiency when compared with fluorescent proteins. 
However, MOLED's also present some drawbacks. As shown in Fig. 14, MOLED's have greater decay times than fluorescent molecules. The time between the moment at which excitation occurs and the moment at which fluorescent proteins reaches 1/e of relative intensity is 2 ns. However, the fluorescence on MOLED's probes last longer, reaching the same value at $20 \mathrm{~ns}$ after excitation. This feature is useful in medical cell imaging, but signifies a disadvantage in the communication field. With enlarged decay time, transmission rate would be lower. Another drawback is the toxicity presented by MOLED's [17], making them inconceivable for medical in-vivo applications. In networks without health risk, it is still a feasible option.

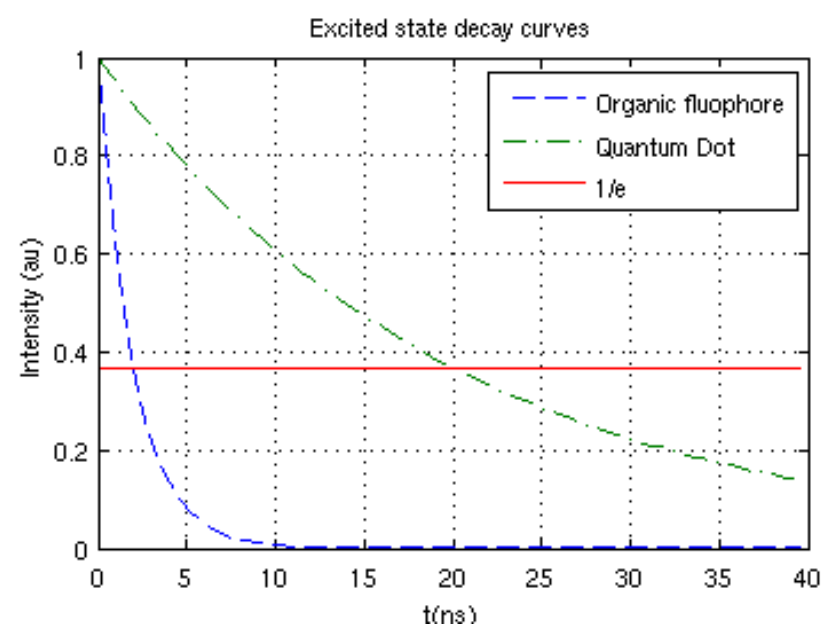

Fig. 16. A comparison of the excited state decay curves between moleds (quantum DOTS) AND COMMON ORGANIC DYES, ACCORDING TO [27].

Although MOLED components currently present the drawbacks mentioned above, its properties could be improved as research on these elements is being in progress. For instance, a novel MOLED using iridium complex phosphor is described in [78]. In comparison with previous MOLEDs and POLEDs (polymer OLED's), this new nano-component has higher efficiency and narrower spectrum width (the emitted power is more concentrated to the expected emission wavelength). 


\subsubsection{Propagation}

In light transduction scenario, the signal is propagated as an electromagnetic wave. As explained in [64], we can deduce the received power from Maxwell's equations:

$$
P_{R}=P_{T} G_{T} G_{R}\left(\frac{\lambda}{4 \pi d}\right)^{2}
$$

where $P_{T}$ is the transmitted power, $G_{T}$ is the antenna gain in emission, $G_{R}$ is the antenna gain in reception, $\lambda$ is the frequency wavelength and $d$ is the distance between transmitter and receptor.

If we are interested in the range of the free space optical communications we can transform equation ( 22 ) to:

$$
d=\frac{\lambda}{4 \pi} \sqrt{\frac{P_{T}}{P_{R}} G_{T} G_{R}}
$$

Thus, if we consider an antenna gain of $3 \mathrm{~dB}$, we expect the received power $-20 \mathrm{~dB}$ of the emitted and a green wavelength $(510 \mathrm{~nm})$, the achievable distance will be:

$$
d=\frac{510 \mathrm{~nm}}{4 \pi} \sqrt{\frac{P_{T}}{10^{-20 / 10} P_{T}} \cdot 2 \cdot 2}=0.812 \mu \mathrm{m}
$$

As it is shown, this is far from our communication expectations. Nano-machines with higher antenna gain and better directivity could be designed to overcome the short optical range. Another possible solution is the usage of semiconductor mixers to convert the optical frequencies to $\mathrm{MHz}$ instead of hundreds of $\mathrm{GHz}$, hence greatly increasing maximum distance propagation. 
Additionally, optical signal could be guided by optical fibers (turning the option to wired scheme) and reach much further distances, by reducing attenuation and scattering. The coverage achievable by this method is enormous compared to other techniques.

\subsubsection{DECODING AND RECEPTION}

Methods to recover the signal at the nano-scale from optical modulation are fundamental in gathering the isolated nano-networks. Once the molecular signal from the emitting nano-network has been translated to optical information, the receiver nano-network requires a technique to convert the signal back to molecular domain. To implement this conversion, molecular wire and molecular switch are proposed. In these techniques, optical waves are converted to guided electrons, constituting an electromagnetic signal. This would require a terminal node with the capability of receiving electrical voltage information, or a pre-converter to molecular signal should be placed before the node. This electron to molecular information conversion is done through synapse processes.

\subsubsection{MOLECULAR WIRE}

The conversion of incoming light signal to molecular information could be developed taking a system inspired in plants photosynthesis methods as a reference.

One possible option is the structure presented in [76], where a molecular wire is described. The proposed wire is 5 molecules length with an optical input. A boron-dipyrromethene dye is used to absorb light, with $62 \%$ absorbed light at $485 \mathrm{~nm}$. The free base porphyrin used for output molecule could be also an option in transmitting part (as an alternative for fluorescent proteins and MOLED's).

Another possible option is the adaptation of light conversion in solar panels, with silicon structure or organic dyes. In [41] and [5], a dye-sensitized ZnO nanowire structure is proposed to transform incoming sun light to electrical energy. After photon capture, electrons are conducted through the wires, which are $16 \mathrm{~nm}$ in diameter. As it is shown in Fig. 15, the nanowire array is the central part of the solar cell, guiding the photons from input light to the platinated cathode, where an electron is released. The same technique could be applied to capture our light incoming signal. 


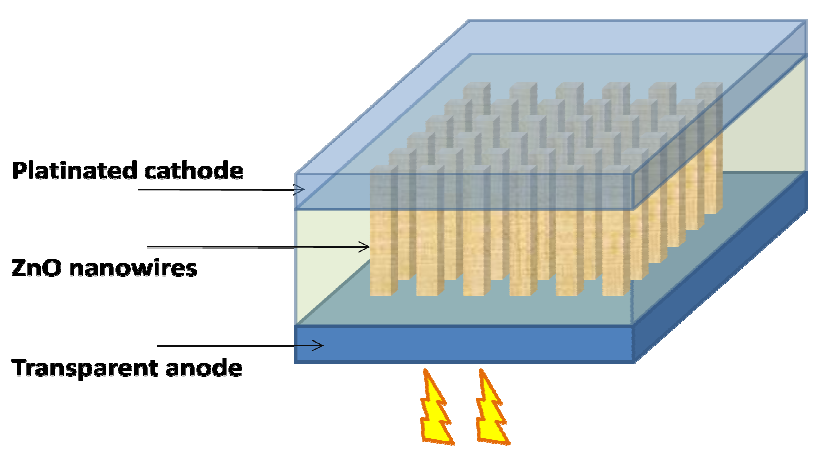

LIGHT

Fig. 17. Nanowire Solar Cell Structure, Described at [41].

\subsubsection{MOLECULAR SWITCH}

A molecular switch is a molecule that can be reversibly shifted between two or more stable states. A subset of molecular switches actuates in response of light variation. The molecular observation of this phenomenon is described in [13].

The light-driven molecular switch implies the physical modification of nanonetwork endpoint. As seen in Fig. 16, the usage of a macromolecule at the endpoint node would provide a physical path when receiving light, while would be in an open circuit configuration in absence of light. Hence, the physical structure can be sensed by detecting whether it is possible a current intensity through the switch.

The molecular switch could implement the adapted filter to decode the incoming optical signal. For example, in a pulse amplitude modulation (PAM), the sensing of the physical structure would give a different logical signal depending on the incoming optical signal. For light presence (e.g., the PAM in high level), there would be some current intensity in the switch, while there would be no possible current for light absence (e.g., PAM in low). 


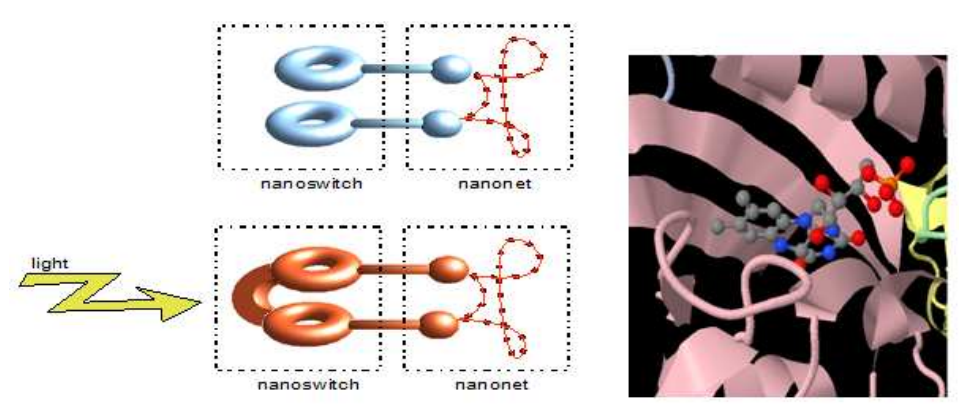

Fig. 18. Left: Physical molecular structure alteration in front of light presence. RIGHT: LOV2 MACROMOLECULE IDENTIFIED AS 1JNU IN RCSB PROTEIN DATABANK ([74]), AS REFERENCED IN [13].

So far, we have covered the wireless options. In the following section we cover the wired ones. Each application peculiarity will force the usage of either wireless or wired techniques, depending on the required features.

\subsubsection{MODEL}

Light transduction is one of the most promising techniques in the interconnection of bio-inspired nano-networks and micro-networks based in silicon semiconductor. The optical interfaces in micro domain have already been developed (using light emitting diodes in micro dimensions). Hence, the optical interfaces to transduct a molecular signal to an optical wave in the nano-domain would enable the usage of light as the common signal understood by nano and micro devices.

\subsubsection{MOLECULAR TO OPTICAL INTERFACES}

Two techniques have been proposed to convert molecular signal to optical information, based on the current research in biomedical cell imaging: fluorescent proteins and bio-compatible quantum dots. Although both of them are capable of implementing the transductor, the most suitable technique for molecular to optical transduction is the bio-compatible nanocrystals, or luminescent quantum dots. These quantum dots offer better optical properties and minimum undesired reactions to other biological components. Among other advantages, quantum dots have higher quantum yields (conversion efficiency), higher photostability and large molar extinction coefficients. 
The main problem of fluorescent proteins utilization as a nano-networks interface is the photo-bleaching. Photobleaching is the photochemical destruction of the fluorophore, the molecular component which causes a molecule to be fluorescent. This phenomenon normally lasts for a single measurement, or signal conversion in our case. For instance, in [72] a Green Fluorescent Protein is measured to have a $40 \%$ bleach depth after $200 \mu \mathrm{s}$. For this, it would be required the fluophores creation or reconstruction in transductor nano-machine. Thus, the complexity of the fluorescent proteins implementation will be much higher than the usage of bio-adapted nanocrystals.

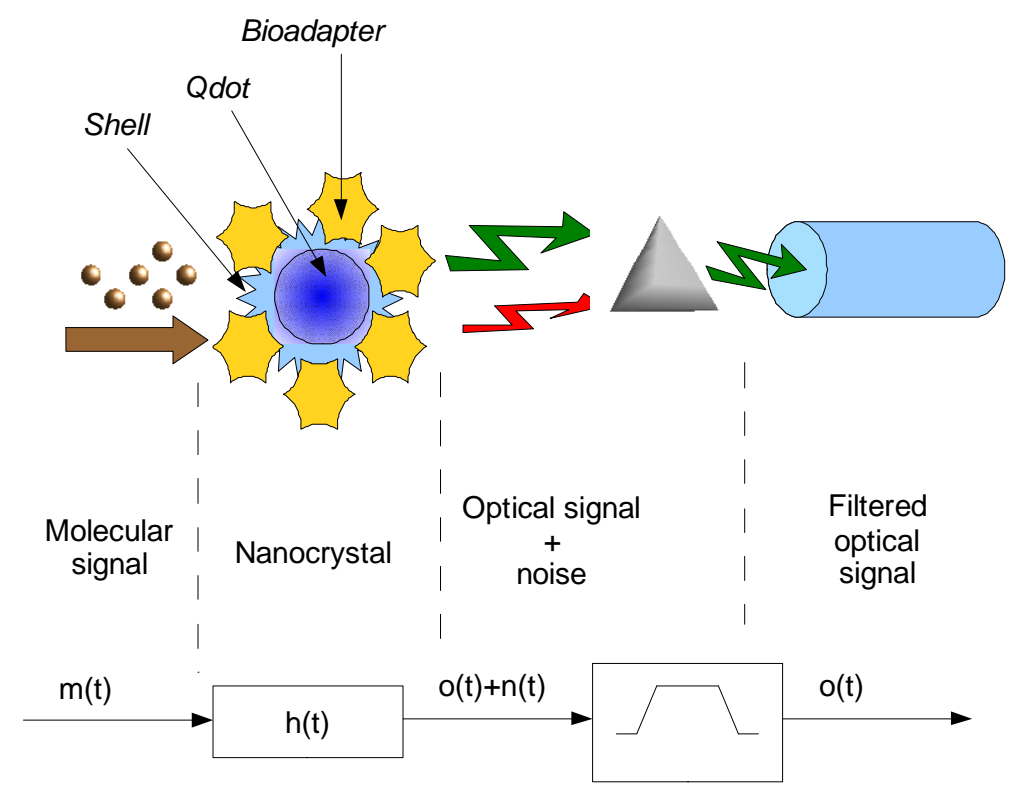

Fig. 19 Molecular to optical CONVERSion SCHEME

The nanocrystals quantum dots are usually a two layer semiconductor structure, namely, the core and the shell. Additionally, the shell can be conjugated with biological components that will make the compound able to detect certain biomolecules (e.g., tumor cells) [27], [26]. The main drawback is the cytotoxicity that quantum dots might present in medical applications. However, as stated in [50], in lower concentrations they are not completely innocuous, but they can accomplish their task without major interference with other biological processes.

The schematic of the conversion between molecular and optical information is shown in Fig. 17. Molecular signal $m(t)$ reaches the bioadapter components around the nanocrystal. Then, the nanocrystal will be able to transmit an optical wave depending whether the presence of the desired molecular signal is detected or not. The emitted optical signal will have undesired 
frequencies that shall be filtered in the transceptor. Finally, the filtered signal can be transported in an optical fiber to reach extremely high distances.

In the detection of molecular signal by the nanocrystal, two methods can be applied: FRET and BRET.

In the Förster Resonance Energy Transfer (FRET) case, the nanocrystal is excited by an external optical signal (around $10 \mathrm{~mW}$ ). If the presence of target cells is detected, the nanocrystal will emit an optical signal in another wavelength. This mechanism is the same that fluorescent molecules use. The FRET mechanism has a donor and an acceptor. The donor is a light-receiving component (e.g., Cyan Fluorescent Protein) that excites the acceptor (e.g., CdSe quantum dot, Yellow Fluorescent Protein). The FRET efficiency is measured by the following equation [75]:

$$
\mu=\frac{1}{1+\left(\frac{r}{R_{0}}\right)^{6}}
$$

where $\mu$ is the FRET efficiency, $r$ is the distance between donor and acceptor and $R_{0}$ is Förster distance. This distance is is typically a few nanometres, depending on the donor and acceptor molecule and their relative orientation. As it can be seen, the distance between donor and acceptor is crucial in FRET efficiency.

Bioluminescence Resonance Energy Transfer (BRET) is a current research topic that could highly improve the features of molecular to optical conversion in our designed transceivers. In BRET conversion no external light is needed, as the donor transmits energy to the acceptor by non-radiative means. BRET transfer has been reported between fluorescent proteins [16] and using nanocrystal. In [65], a BRET mechanism in CdSe/ZnS nanocrystal is studied. A mutation of the $R$. reniformis luciferase (Luc8) is used as a biological component that triggers quantum dot emission. The resulting emitted optical signal has a component in $480 \mathrm{~nm}$ corresponding to Luc8, but a bigger peaks are found in wavelength from $600 \mathrm{~nm}$ to $800 \mathrm{~nm}$ (depending on the used nanocrystal). 
Quantum dot emitters based on BRET transmission is the most promising technique for two main reasons. First of all, the optical wave is transmitted only when there is the molecular signal, instead of sensing the molecular signal with an external laser. Although it would imply an asynchronous behavior (as the transceiver do not know when the incoming signal will arrive), the channel occupation can be dramatically reduced (as the periodic sensing from the transceiver disappear) and there is no need of molecular signal buffering (that would be necessary in FRET while waiting for incoming sensing signal). Secondly, the power required to achieve a BRET transmission is much lower than the power needed for FRET. As an example, in [68] it is compared the energy needed via BRET versus external excitation of $1 \mu \mathrm{M}$ of the conjugated quantum dot under $1 \mathrm{~cm}$ of tissue (in $1 \mu \mathrm{L}$ ).

$$
E_{B R E T}=\phi \times n \times C \times V \times h v=\phi \times n \times V \times \frac{c}{\lambda} \times h
$$

where $\phi$ is the flux of exciting photons in phot $/ \mathrm{s} / \mathrm{mol}, n$ is the number of luciferase molecules per quantum dot, $C$ is the concentration of quantum dots in $M / I, V$ is the volume in litres, and $h v$ is the energy of a photon.

In our case, under saturating conditions, $3.6 \times 10^{10}$ photons of $\lambda=655 \mathrm{~nm}$ will be produced per second. The total energy required for this emission comes from the conjugated Luc8's, at $\lambda=480 \mathrm{~nm}$. Applying equation ( 26$)$ to our case $(1 \mu \mathrm{M}$ of quantum dot conjugates, $1 \mu \mathrm{L}$ in volume), we obtain:

$$
E_{B R E T}=1.5 \cdot 10^{22} \times 6 \times 1 \cdot 10^{-6} \times 1 \cdot 10^{-6} \times \frac{3 \cdot 10^{8}}{480 \cdot 10^{-9}} \times 6.6 \cdot 10^{-34}=37 \mathrm{nW}
$$

An to produce the same quantum dot emission by external excitation:

$$
E_{F R E T}=\frac{\phi^{\prime}}{\mu \times K} \times A \times h v
$$


where $\phi^{\prime}$ is the expected flux of photons in phot/s, $\mu$ is the quantum yield (efficiency) of the nanocrystals, $K$ is the absorption (related to extinction coefficient), $A$ is the attenuation of the tissue, and $h v$ is the energy of a photon.

To have the same amount of photons than in the BRET case, the energy in FRET case would be:

$$
E_{F R E T}=\frac{3.6 \cdot 10^{10}}{0.83 \times 0.5} \times 2.6 \cdot 10^{8} \times \frac{3 \cdot 10^{8}}{480 \cdot 10^{-9}} \times 6.6 \cdot 10^{-34}=9.3 \mathrm{~W}
$$

\subsubsection{OPTICAL TO MOLECULAR INTERFACES}

The most promising technique to convert optical information to a molecular signal is the usage of optical molecular switches. Instead of obtaining electrons from the received photons (as it is the case of molecular wires, dyes in photo devices and advanced solar cells), the structure of molecular switches changes in function of the received light. Depending on the absence or presence of the optical signal, the molecular circuit will present a different structure. The implementation of molecular switches is more direct than the other cited options (e.g., molecular wire) and can be mapped in micro semiconductor terms. As it can be seen in Fig. 18, the optical molecular switch could be the key component in the receptor nanomachine. In the figure, the electrical (or molecular) signal $x_{O}(t)$ would have $V$ amplitude or null amplitude depending on the incoming optical signal $x_{i}(t)$.

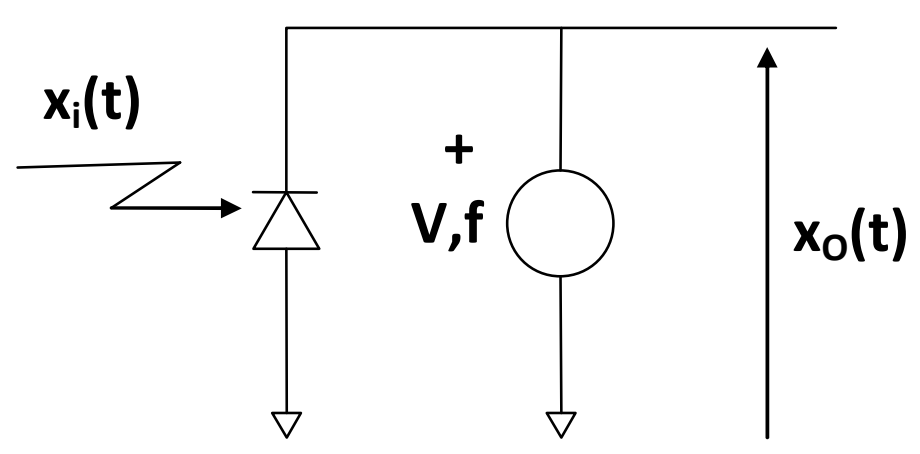

Fig. 20 ELECTRICAL MOdEL OF A POSSIBLE IMPLEMENTATION OF AN OPTICAL TO MOLECULAR SIGNAL INTERFACE USING AN OPTICAL MOLECULAR SWITCH AS A TRANSDUCER. 
The current in the optical molecular switch can be, according to [80], calculated with the Laundauer-Bütiker equation:

$$
\begin{gathered}
I(V)=G_{0} \int n(E) T(E, V) d E \\
G_{0}=\frac{2 e^{2}}{h}
\end{gathered}
$$

where $G_{0}$ is the quantum unit of conductande, $h$ is the Plank's constant, $n(E)$ is the distribution function, and $T(E, V)$ is the transmission coefficient for electrons with energy $E$ for voltage $V$. The distribution function can be expressed using the following equation:

$$
n(E)=f\left(E-\mu_{L}\right)-f\left(E-\mu_{R}\right)
$$

where $f$ is the Fermi function, $\mu_{L}$ is the electrochemical potential of the left electrode and $\mu_{R}$ is the electrochemical potential of the right electrode.

Using equations ( 30$)-(32)$ the current intensity through the molecular switch can be visualized for different voltage in Fig. 19. In the figure, the red line is the current in open-ring configuration, whereas the black line is the current in closed-ring structure. As it can be seen in the figure, for 1 Volt applied in the molecular switch, the current for the different structure (depending on whether the optical signal is irradiating the switch or not) is separated by several micro-amperes. 


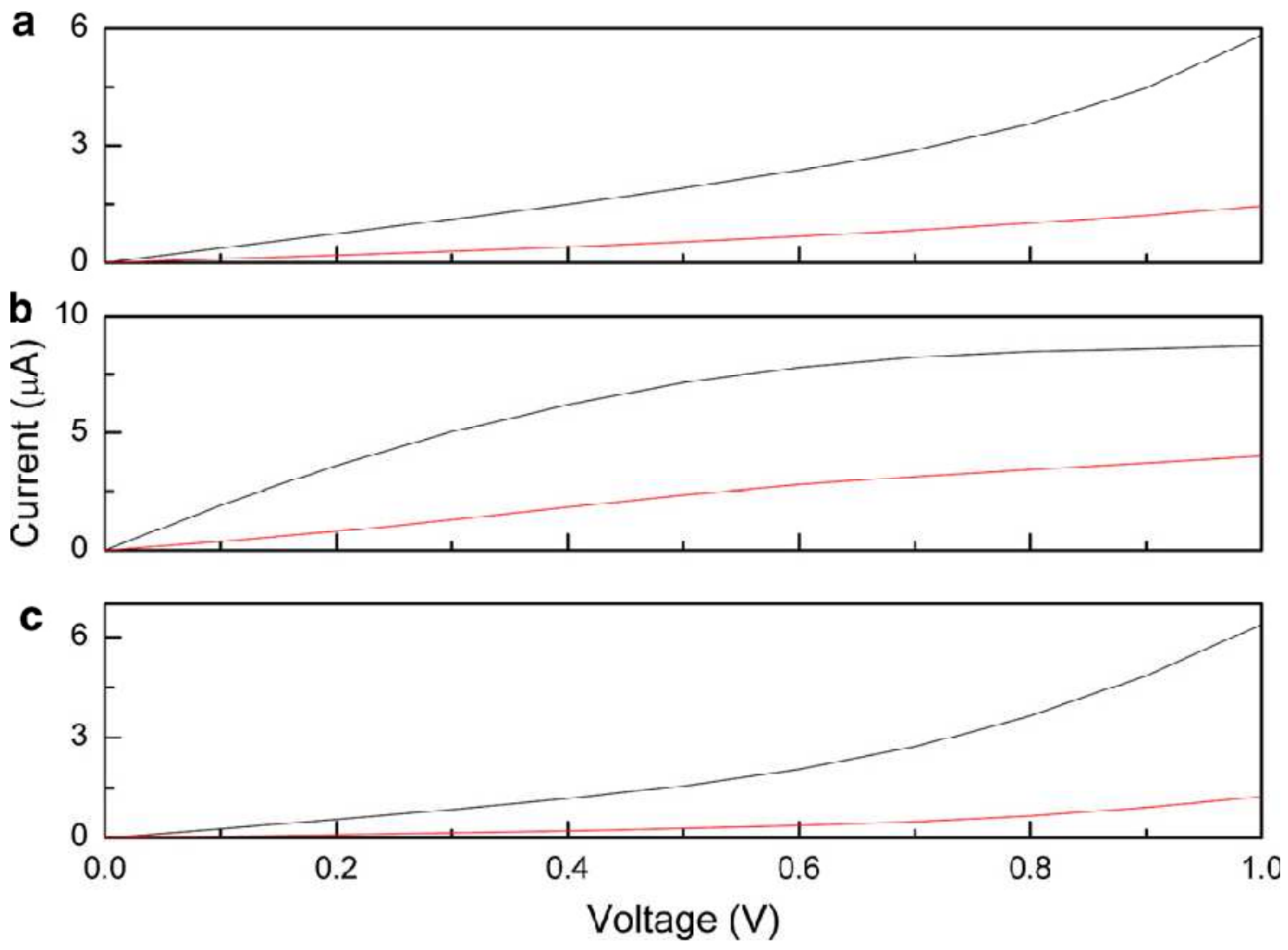

Fig. 21 CURRent - Voltage CURVES IN THE MOLECULAR SWITCH, FOR 3 Different STRUCTURES OF MOLECULAR CONTACTS ( $A=$ HOLLOW, B=TOP, C=BRIDGE). 


\section{WIRED OPTIONS}

Wired molecular communication can be realized by means of a physical link for signal propagation. In the following subsections, two different types of molecular physical links are proposed, namely, axons (explained in subsection A) and capillaries (explained in subsection B).

\subsection{AxONS AND ACTION POTENTIAL}

The nerve fibers that animal brain uses to order muscle movements, the intra-body transport of external sensorial stimulus and the neural communication in the animal brain are the underlying concepts on which this technique is inspired. Axons, the slender projection of the neuron, offer promising features for nano-communication.

\subsubsection{ENCODING AND EMISSION}

The signal travelling along an axon is an electrical impulse, namely action potential. In encoding and emission stage, a method to transform the molecular information coming from short range molecular networks (e.g., calcium ions) to action potential electrical signal is clearly needed. The conversion in biological world is done by synapse processes, most of them chemical synapses, through specialized junctions that communicate nerve or neuronal cells with non-neuronal cells. In the human brain there are approximately 1 billion $\left(10^{9}\right)$ synapses for each $\mathrm{mm}^{3}$ [3].

\subsubsection{PROPAGATION}

As it has been previously mentioned, the signal traveling along the axon is the action potential. This unidirectional signal is an electrical pulse around $80 \mathrm{mV}$ in amplitude, rising from a rest potential around $-40 \mathrm{mV}$. Amplitude, rest potential and recovery time vary slightly between different species. For instance, in Fig. 20 the action potential of a giant squid is represented, according to [56]. Its rest potential is $-45 \mathrm{mV}$ and the action potential spike reaches $+40 \mathrm{mV}$. The pulse lasts for $0.5 \mathrm{~ms}$ approx., and the recovery time is $2 \mathrm{~ms}$ approx. After action potential peak, cells constituting the axon re-equilibrate the molecular electrical charge by opening certain ion gates. 


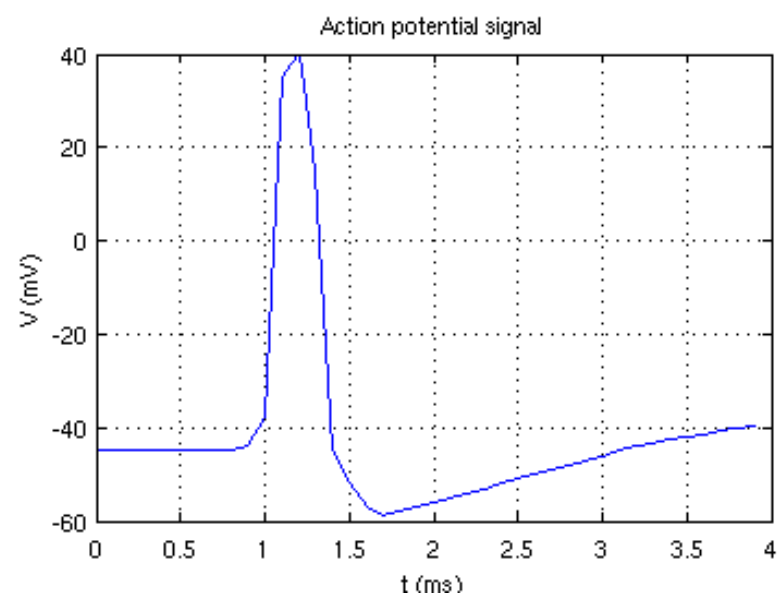

Fig. 22. ACtion Potential WAVEForm of A Giant SQUid, ACCORDing to [56].

Communication through action potential on axons can provide several advantages to our system:

i) Long length. Axon length is large, in nano-scale terms. According to [23], axons can be up to 1 meter, as it is the case of the human nerve that goes from the spinal cord down to the toe. Moreover, the connection of several neurons is already implemented in biological domain using the synapse process. This process is a chemical interaction between the neuron dendrite and axons terminal (see Fig. 21 for neuron structure). Despite of the speed reduction that would imply several synapse processes (as it is the bottleneck process in neuronal communication), the distance coverage that could be achieved should be taken into account.

ii) High signal speed. As stated in [35], this is determined by axon radius and the presence of myelin sheath. In myelinated largest axons, the action potential is able to reach up to 90 $\mathrm{m} / \mathrm{s}$. Myelin is composed of 80 percent lipid and 20 percent protein and turns axons in a type of coaxial cable. Myelin wraps around the axon and very efficiently insulates it, improving energy usage.

iii) Very low attenuation. The attenuation is very low in animal nerve axon. As it is stated in [23], the neural impulse does not weaken while travelling along the axon.

iv) Electrical signal. Action potential is an electric signal, thus enabling semiconductor receivers and transmitters to be attached directly to the axons. This would constitute an interconnection path between different nanonetworking techniques. Furthermore, Ranvier nodes could be used as plug interface. Ranvier nodes are the spaces between the myelin 
sheaths, approximately 1 micrometer in length. At nodes of Ranvier, the axonal membrane is uninsulated and therefore capable of generating electrical activity (see Fig. 21 for neuron structure).

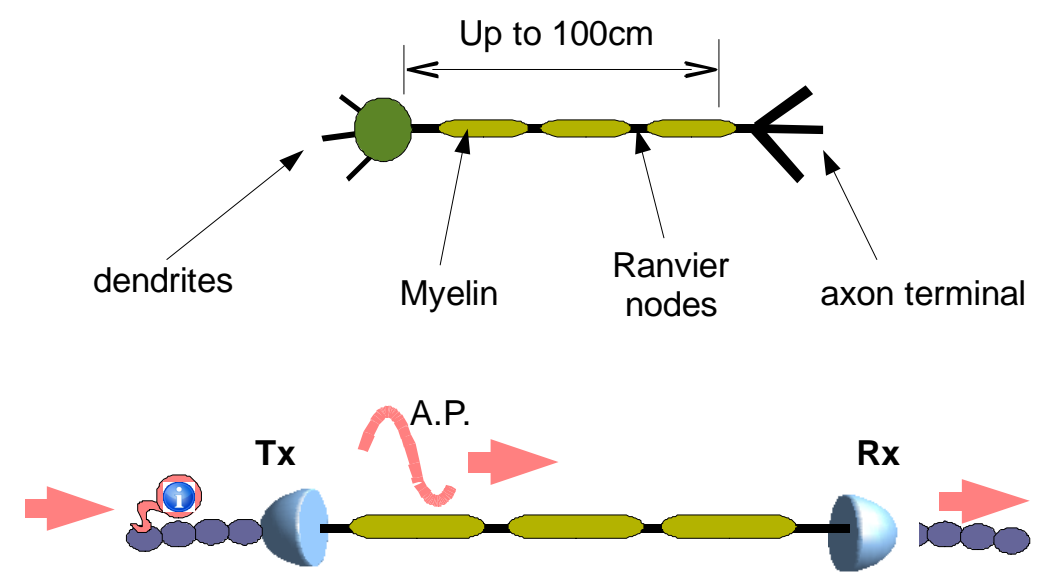

Fig. 23. Top: Structure of a neUron. Bottom: axons possible use to transmit action POTENTIAL IMPULSES BETWEEN NANONETWORKS NODES.

\subsubsection{DECODING AND RECEPTION}

The reception of action potential can be conformed back to molecular information by synaptic processes. In neuronal communication, the action potential is received through the dendrites, and can be passed to other neurons by the axon terminals. The pre-synaptic part stores several chemical molecules, namely neuro-transmitters, which are released when action potential is received. In the post-synaptic part, these neuro-transmitters will be received through specialized junctions and will trigger a new action potential (or would be converted into a different molecular signal for our nano-machine).

The conversion between the molecular signal and the action potential impulse is the slowest of all the processes involved in neuronal communications. The theoretical bandwidth limit of a single axon is around $333 \mathrm{bps}$, as only one action potential peak is able to be transmitted each $3 \mathrm{~ms}$ ( $0.5 \mathrm{~ms}$ of action potential pulse, plus $2.5 \mathrm{~ms}$ of cell recovering period).

\subsubsection{MODEL}

As stated in the previous section, the slowest stage in long range communication using axons as a physical support is the conversion between the molecular signal and the electrical impulse of action potential (the signal traveling along the axon). Hence, this process will be the target of the axon modeling. 
The conversion between molecular and electrical information is realized through the chemical synapse process. As it is shown in Fig. 22, the conversion from electrical action potential (A.P.) to a molecular signal is realized in the extreme of the neuron, namely, the axon terminal, where action potential triggers the release of molecular neurotransmitters (m.T.) in a region called pre-synapse (Pre.Syn.). The released neurotransmitters are able to be bound in the post-synapse receptors, where they can be converted to other molecular signals or trigger another action potential. In Fig. $\mathbf{2 3}$ the process to convert molecular signal to action potential is depicted. Through a certain ion gates, the adequate molecules can enter to the dendrites of next neuron, triggering the A.P. electrical impulse that travels through its axon.

For the modeling of the synapse we will discuss the following models:

- HODS model [47]. Focusing on pre-synapse. This model is an improvement of the following previously defined models:

- Dynamic synapse model [44]. This model additively models presynaptic effects on the postsynaptic response.

- Facilitation-depression model [20]. This model is a fundamental multiplicative model in which the multiplicative product of presynaptic activities is applied to affect the postsynapse.

- Kinetic Binding model [18]. Focusing on post-synapse. This model allows a realistic biophysical representation, based on the post-synaptic receptor binding.

- Information Theory model [48]. This model describes synaptic transmission in an information-theoretic perspective.

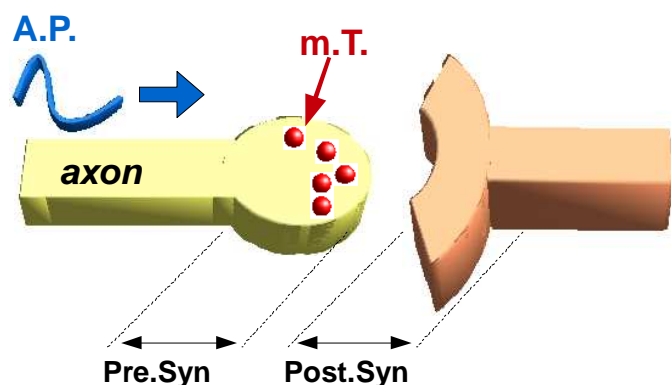

FIg. 24 SyNAPSE PROCESS TO CONVERT THE ACTION POTENTIAL TO A MOLECULAR SIGNAL.

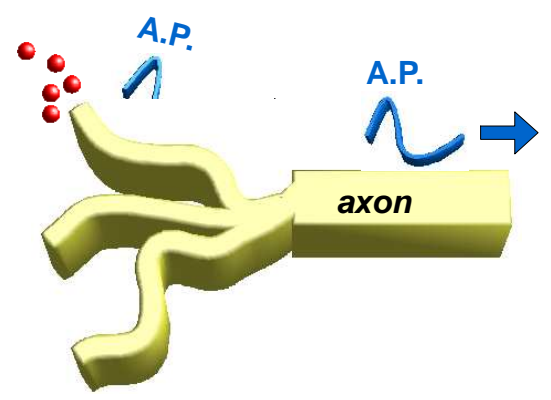

FIG. 25 SyNAPSE PROCESS tO CONVERT THE MOLECULAR SIGNAL TO ACTION POTENTIAL. 


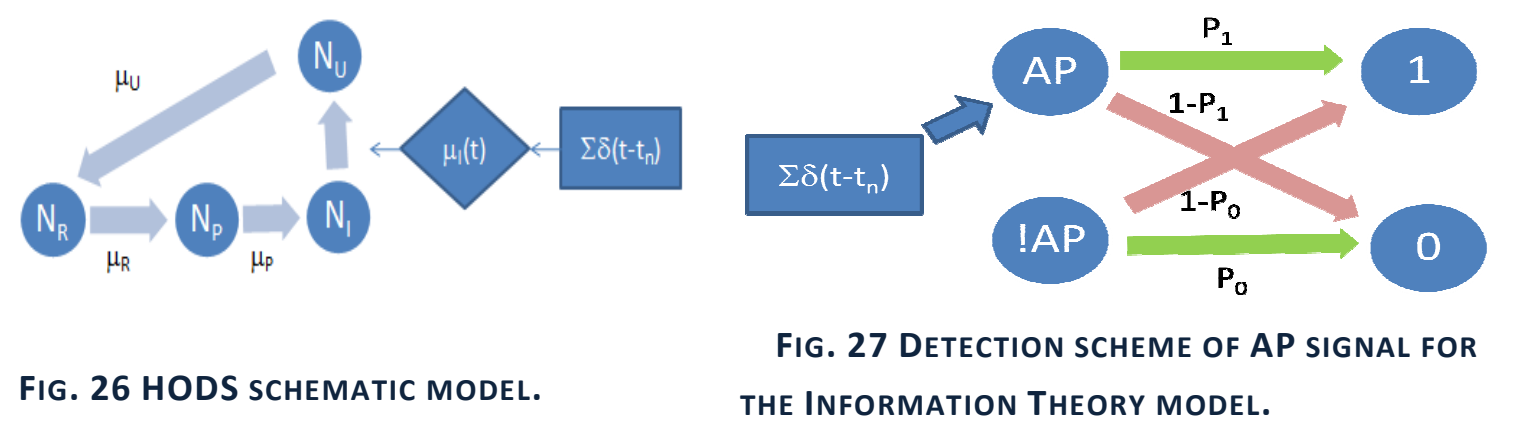

\subsubsection{HODS MODEL}

The HODS model stands for nonlinear High-Order Model for Dynamic Synapse. In this model, the neurotransmitters in pre-synaptical part are assumed to be in different vesicle pools. As it is shown in Fig. 24, the vesicle pools are classified in 4 different types, according to its function and its position in the membrane:

$N_{l}$ : number of vesicles in the immediate release pool. When Action Potential impulse is recieved, the neurotransmitters in this pool are released to the cleft (the space between pre-sinaptical and post-sinaptical part).

$N_{P}$ : number of vesicles in prepared release pool. Its function is to refill the immediate release pool when the neurotransmitters are released to the cleft.

$N_{R}$ : number of vesicles in the reserve pool. The reserve pool refills the vesicles of the prepared release pool.

$N_{U}$ : the used pool collects used neurotransmitters after synaptical process, to be re-used for future sinapse processes.

The constant parameters $\mu_{R}, \mu_{P}$ and $\mu_{U}$ represent the transfer rates between neighboring pools (Fig. 24) and $\mu_{l}(t)$ is the nonstationary release rate which is a function of input pulses $\sum_{n} \delta\left(t-t_{n}\right)$. The probability of transfer between pools is considered a binomial variable probability, converging to a Poisson distribution for a high number of vesicles in each pool. In steady state, the four pools are described in [47] by the following equations: 


$$
\begin{gathered}
\frac{d N_{R}(t)}{d t}=-\mu_{R} N_{R}(t)+\mu_{U} N_{U}(t) \\
\frac{d N_{P}(t)}{d t}=-\mu_{P} N_{P}(t)+\mu_{R} N_{R}(t) \\
\frac{d N_{I}(t)}{d t}=\mu_{P} N_{P}(t)-\mu_{I} N_{I}(t) \sum_{n} \delta\left(t-t_{n}\right) \\
\frac{d N_{U}(t)}{d t}=-\mu_{U} N_{U}(t)+\mu_{I} N_{I}(t) \sum_{n} \delta\left(t-t_{n}\right)
\end{gathered}
$$

By concatenatively repplacing the variables in equations ( 33 ) - ( 36 ) while preserving the variables $\mu_{l}(t)$ and $N_{l}(t)$, the synaptic activities can be written as the following third-order differential equation:

$$
\begin{gathered}
K_{3}\left[\frac{d^{3} N_{I}}{d t^{3}}+\frac{d^{2}\left(\mu_{I} N_{I}\right)}{d t^{2}} A P\right]+K_{2}\left[\frac{d^{2} N_{I}}{d t^{2}}+\frac{d\left(\mu_{I} N_{I}\right)}{d t} A P\right]+K_{1}\left[\frac{d N_{I}}{d t^{2}}+\mu_{I} N_{I} A P\right]+N_{I} \\
=0
\end{gathered}
$$

where

$$
\begin{gathered}
K_{3}=\frac{1}{\mu_{U} \mu_{R} \mu_{p}} \\
K_{2}=\left(\frac{1}{\mu_{U} \mu_{R}}+\frac{1}{\mu_{R} \mu_{P}}+\frac{1}{\mu_{U} \mu_{P}}\right) \\
K_{1}=\left(\frac{1}{\mu_{U}}+\frac{1}{\mu_{U}}+\frac{1}{\mu_{U}}\right) \\
A P=\sum_{n} \delta\left(t-t_{n}\right)
\end{gathered}
$$


In our nanocommunication perspective, the relation between the number of vesicles in the immediate release pool and the Action Potential impulse will give us the transfer function of the synapse process.

\subsubsection{KINETIC BINDING MODEL}

In [18], a simple kinetic synapse model is described based on reasonable biophysical assumptions. Hence, the system behavior can be defined by the following equation:

$$
\frac{d r}{d t}=\alpha[T](1-r)-\beta r
$$

where $r$ is the percentage of bound receptors, $T$ is the number of neurotransmitters in the mediu and $\alpha$ and $\beta$ are parameters indicating the binding/release affinity of the post-synaptic receptors.

The action potential is associated to the release of neurotransmitter molecules. When the electrical signal (Action Potential) reaches the pre-synaptic part, $1 \mathrm{mM}$ of neurotransmitters are released. The process lasts for $1 \mathrm{~ms}$, in which the receptors $r$ in the post-synaptical part are able to capture the released molecules. After this time, the neuromolecules present in the cleft (space between pre-synapse and post-synapse regions) decrease abruptly. This behavior is modeled as a pulse waveform of the neurotransmitters $T$, thus providing a easy analytical solution to equation ( 42 ), splitting the expression for $T(t)=T$ and for $T(t)=0$. Considering a pulse between $t_{0}$ and $t_{1}$, the equation ( 43 ) defines the receptors evolution during the pulse, and the equation ( 44 ) expose the receptor relaxation after the pulse.

$$
\begin{gathered}
r\left(t-t_{0}\right)=\frac{\alpha T}{\alpha T+\beta}+\left(r\left(t_{0}\right)-\frac{\alpha T}{\alpha T+\beta}\right) e^{\frac{-\left(t-t_{0}\right)}{\tau_{r}}} \\
r\left(t-t_{1}\right)=r\left(t_{1}\right) e^{-\beta\left(t-t_{1}\right)}
\end{gathered}
$$

where 


$$
\tau_{r}=\frac{1}{\alpha T+\beta}
$$

As it is shown in Fig. 26, the receptors will bind following an exponential fashion, emulating an electrical condenser model. In that case, the action potential pulses are separated $4 \mathrm{~ms}$. Assuming that the maximum number of bound receptors for detection of a logical " 0 " is $10 \%$, this would imply a maximum information transmission of about $1 \mathrm{bit} / 4 \mathrm{~ms}=250 \mathrm{~Hz}$. As it can be seen in Fig. 27, increasing the frequency rate of action potentials implies a higher level of bound receptors in absence of AP signal.

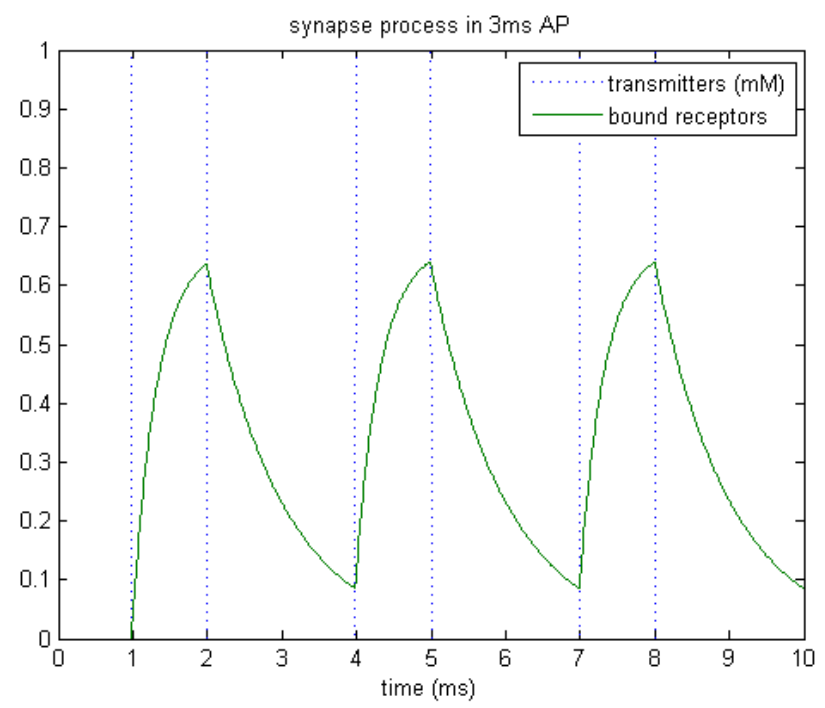

Fig. 28 KINETIC MODEL, IMPULSES EACH 4MS. 


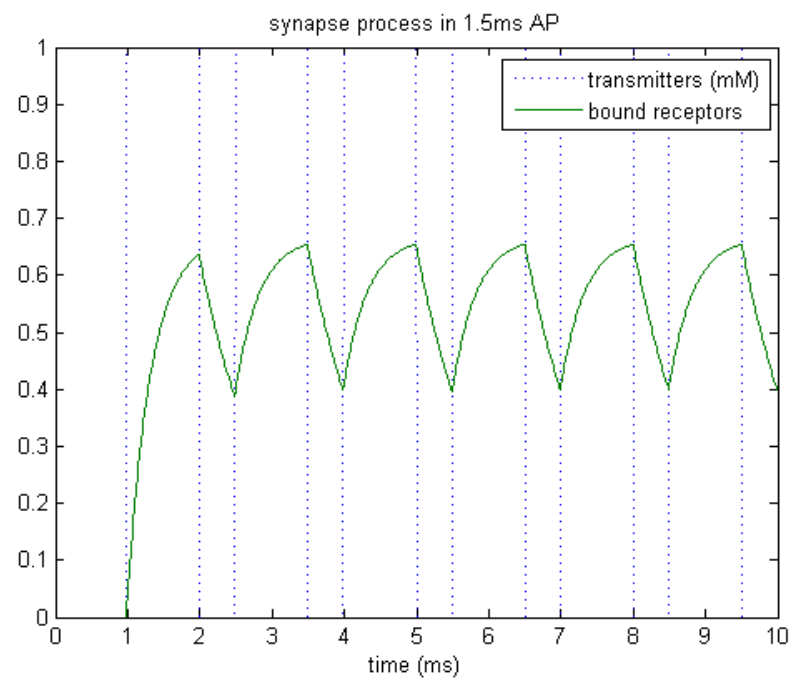

Fig. 29 KINETIC MODEL, IMPULSES EACH 1.5MS.

\subsubsection{INFORMATION THEORY MODEL}

The synapse process under an information theory point of view has been studied in [48]. In this paper, the following equation is proposed to find the mutual information that can be achieved for a single synapse:

$$
I_{l b}=\frac{1}{2} \int_{S} \log _{2}\left[\frac{S_{m m}(\omega)}{S_{\hat{n} \hat{n}}(\omega)}\right] d \omega
$$

where $S_{m m}(\omega)$ is the power spectral density of the stimulus $m(t)$, and $S_{\hat{n} \hat{n}}(\omega)$ is the power of the reconstruction noise $\hat{n}(t)=\widehat{m}(t)-m(t)$.

Although in the referenced article it is proposed multiple synapses to add redundancy and increase the information throughput, a single synapse process would be able to transmit about $4 \mathrm{bits} / 200 \mathrm{~Hz}$.

\subsection{CAPILLARIES}

Capillaries are the smallest of blood vessels, measuring from $5 \mu \mathrm{m}$ to $10 \mu \mathrm{m}$ in diameter. They connect arterioles and venules and their main function is to interchange chemicals and 
nutrients between blood and surrounding tissues. The length of capillaries is comparable to axons length. In [39], capillaries measuring $67 \mathrm{~cm}$ long are used as testing units. In addition to this, several capillaries can be joined, reaching further nodes. In Fig. 30, a possible architecture using capillary tubules is shown. In that figure, node 1 would release the particles into the medium. Traveling into the flow, the message would reach node 2, equipped with receptors able to capture the released particles.

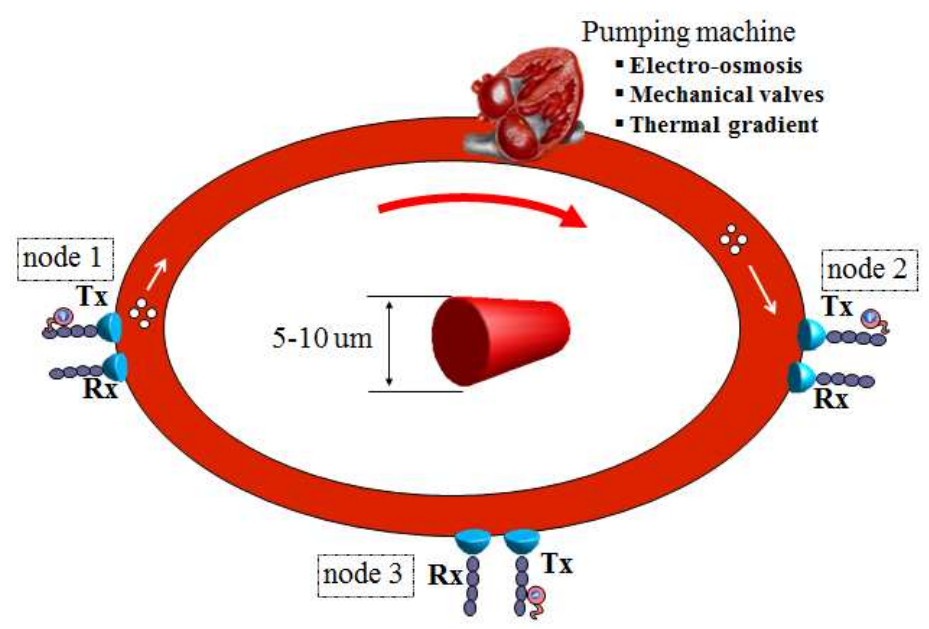

FIG. 30 CAPILLARY CIRCUIT IN A TOKEN RING IMPLEMENTATION EXAMPLE

\subsubsection{ENCODING AND EMISSION}

The emulation of capillaries for long-range wired architectures enables the usage of most of communication particles that are already used in animal blood. In the capillary circuit, nodes would have to sense the flow current and load packets (particles or molecules) using appropriate binding receptors, or release particles to transmit the information. Hormones are the most suitable particles to be used. Like pheromones in the wireless case, different types of hormones exist in nature. Additionally, hormones are able to bind only to its particular receptor, thus providing a built-in selective filter. Endocrine hormones, which travel in the blood stream, serve as chemical messenger to communicate cells inside the body. Of a particular interest is epinephrine, a hormone that triggers the flight or fight response: in front of a threat, the animals have to face the menace or leave the scene as fast as possible. As it is a type of alarm system, the release and bind process have to be very fast, enhancing the performance of designed nanonetwork. According to [12] 30,000 molecules should be released to correctly recover a signal peak consisting of 5 components. 


\subsubsection{PROPAGATION}

The propagation of molecular particles inside the capillaries is governed by fluid mechanics. In fluid mechanics, the Reynolds number $R e$ is a dimensionless number that characterizes different flow regimes, e.g., laminar or turbulent flow. In laminar flow (low Reynolds number), viscous forces are dominant and the fluid motion is smooth and constant. In turbulent flow (high Reynolds number), the inertial forces dominate and tend to produce flow fluctuations. Reynolds number $R e$ in a pipe is defined as [8]:

$$
R e=\frac{\rho v r}{\mu}
$$

where $\rho$ is the density of the fluid $\left(\mathrm{kg} \cdot \mathrm{m}^{-3}\right), v$ is the velocity of the fluid $(\mathrm{m} / \mathrm{s}), r$ is the radius of the capillary $(\mathrm{m})$ and $\mu$ is the dynamic viscosity of the fluid (Pa.s).

Capillary circuit is in the low Reynolds number regime. Taking a value for fluid viscosity $\mu=2$ $\mathrm{mPa} \cdot \mathrm{s}$ (plasma, [31]), a pure water density of $\rho=1000 \mathrm{~kg} / \mathrm{m}^{3}$, a radius of $r=2.5 \mu \mathrm{m}$ and a fluid velocity of $v=1 \mathrm{~cm} / \mathrm{s}$, the Reynolds number would be $R e=0.0125$ " 1 . In this regime, viscous effects dominate the dynamics and inertial effects, which cause turbulence, are negligible.

The hardware required to use capillary tubules as guided propagation is complex and has to be carefully monitored. Special attention has to be dedicated to flow current generation and capillary integrity. The pumping mechanism is a key factor in capillary communication. In [15], fluid propulsion based on electro-osmosis is proposed. The main advantage of this technique is its independent implementation, allowing the pumping coupling to be included in the circuit without altering the general structure. However, the extremely high electrical fields required for its implementation is a major drawback. Other alternatives are a thermal circuit (using a temperature gradient to induce the fluid movement), or biological valves and mechanical micro-pumps.

\subsubsection{Decoding And Reception}

Reception in capillaries circuit relies on binding affinities of the used particles (hormones). In nature, epinephrine receptors are the adrenergic receptors, capable of translating epinephrine signal to muscle movement. However, the antagonists (unexpected particles that bind to the 
receptor) are an important noise source depending on receptor subtype. The antagonist amount present in capillary circuit (or the probability of its presence) is the main characteristic to choose between adrenergic receptor subgroup. In example, if Butoxamine could be found in the flow, $\beta_{2}$ subgroup receptor should be discarded.

\subsubsection{MODEL}

Once the molecules are being propagated in capillary tubules, their detection probability depends on what is called the receptor/ligand binding theory. Simply stated, the membrane of a cell contains hundreds, or even thousands, of diminutive receptors to which these molecules may bind. We can define this formally as follows:

A ligand is a substance that is able to bind and form a complex with a biomolecule to serve a biological purpose.

A receptor is a protein molecule, embedded in either the plasma membrane or the cytoplasm of a cell, to which a molecule used for signalling may attach.

Once the ligand is bound to a receptor, the new structure is a complex, which will keep the ligand bounded depending on specific cell related constants $\left(k_{f}\right.$ and $\left.k_{r}\right)$.

In [45], the ligand-binding theory is analyzed on basis to the following formula:

$$
\frac{d C(t)}{d t}=k_{f} R(t) L(t)-k_{r} C(t)
$$

where $C$ is the number of complex molecules, $R$ is the number of receptors in the cell, $L$ is the concentration of ligands (in $\mathrm{M}$ ) and $k_{f}$ and $k_{r}$ are constants whose value depends on receptor cell type.

As in [45], we restrict our attention to situations in which the cohort of surface receptors is unchanged, thus fixing total receptor number to constant amount $\mathrm{R}_{\mathrm{T}}$. However, in the referenced paper the simplification is also applied to $L(t)$ (considering fixed the ligand concentration) and it is unacceptable for our purposes, as our information will be encoded in ligand concentration fluctuation. 


$$
\begin{gathered}
R(t)=R_{T}-C(t) \\
L(t)=A \sin (2 \pi f t)+L_{o} \\
\frac{d C(t)}{d t}=k_{f} R_{T} L(t)-\left(K_{f} L(t)+K_{r}\right) C(t)
\end{gathered}
$$

To determine Lo (to which our signal will be added), we will compute the equilibrium of complexes. When reaching that equilibrium, no further variation should be applied, thus obtaining $\frac{d C(t)}{d t}=0$. Then, equation ( 51 ) becomes:

$$
\begin{gathered}
0=k_{f} R_{T} L_{o}-\left(K_{f} L_{o}+K_{r}\right) C_{e q} \\
C_{e q}=\frac{k_{f} R_{T} L_{o}}{k_{f} L_{o}+k_{r}} \underset{K_{D}=k_{r} / k_{f}}{\longrightarrow} C_{e q}=\frac{R_{T} L_{o}}{L_{o}+K_{D}}
\end{gathered}
$$

where $K_{D}$ is the dissociation constant. Assigning a $L o$ value of $K_{D}$, the number of complexes in equilibrium is $R_{T} / 2$. In other words, the receptor steady-state is reached when half of its receptors are occupied.

To run the numerical analysis, we will sample the evolution of complexes quantity (number of bound receptors). If we assume that the sampling time $\Delta t$ obeys the Nyquist-Shannon sampling constraint:

$$
\Delta t<\frac{1}{2 B_{w}}
$$

then there is no loss of the information coming from the transmitter when the signal $C(t)$ is being sampled. $B_{w}$ is the bandwidth of the Fourier transform before the sampling. In our case, 
we will introduce fluctuations up to $250 \mathrm{~Hz}$, hence the time increment must be smaller than $2 \mathrm{~ms}$.

If we discretize the equation ( 51 ) we will have:

$$
\begin{gathered}
\Delta C(t)=\left\{K_{f} R_{T} L(t)-\left(K_{f} L(t)+K_{r}\right) C(t)\right\} \Delta t \\
C[n+1]=C[n]+k_{f} R_{T} L[n] \Delta t-\left(K_{f} L[n]+K_{r}\right) C[n] \Delta t
\end{gathered}
$$

To establish the receptor parameters, we will compare an alpha adrenergic receptor (in charge of adrenaline receiving in living organisms) and a light chain interleukin (IL). The following parameters, taken from [45], are used in our simulation.

\begin{tabular}{ccc}
\hline & $\alpha$-Adrenergic & Interleukin \\
\hline cell type & BC3H1 & T lymphocytes \\
$\boldsymbol{R}_{T}(\# /$ cell $)$ & $1.4 \times 10^{4}$ & $1.1 \times 10^{4}$ \\
$\boldsymbol{k}_{\boldsymbol{f}}\left(\boldsymbol{M}^{-\mathbf{1}} \mathbf{m i n}^{-1}\right)$ & $2.4 \times 10^{8}$ & $8.4 \times 10^{8}$ \\
$\boldsymbol{K}_{\boldsymbol{r}}\left(\mathbf{m i n}^{-1}\right)$ & 0.018 & 24 \\
$\boldsymbol{K}_{\boldsymbol{D}}$ & $7.5 \times 10^{-11}$ & $2.9 \times 10^{-8}$ \\
\hline
\end{tabular}

TABLE 1 Different PARAMETERS OF SIMULATED RECEPTORS

As it is shown in Fig. 31, adrenergic receptor is unable to provide an acceptable response to ligand frequency variation. 

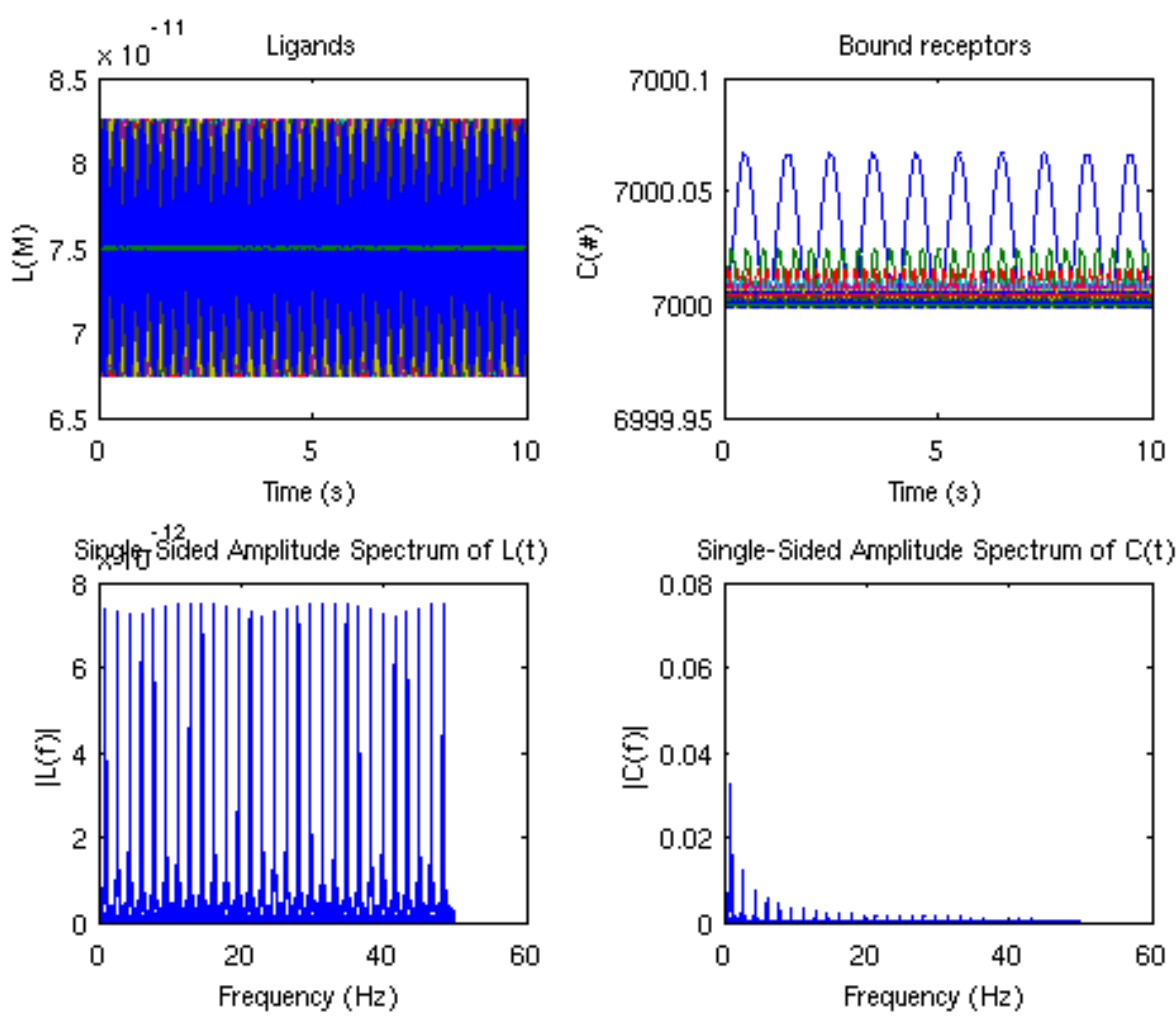

FIG. $31 \alpha$-ADRENERGIC RECEPTOR SIMULATION. TOP-LEFT: INPUT SIGNAL AS A SINUSOID VARIATION IN LIGAND CONCENTRATION. TOP-RIGHT: NUMBER OF LIGANDS THAT HAVE BEEN CAPTURED BY RECEPTORS. BOTTOM-LEFT: SPECTRAL ANALYSIS OF INPUT SIGNALS (LIGANDS). BOTTOM-RIGHT: SPECTRAL ANALYSIS FOR BOUND RECEPTORS (COMPLEXES)

This can be explained for its low level of $K_{r}$ parameter, which reflects the tendency of the receptor to keep the ligand molecule in the complex form (bound to the receptor). As $K_{r}$ is low in adrenergic receptors, the system cannot discharge fast enough to follow the sinus function in the input signal (the ligand concentration). Indeed, we can visualize the decay curve of complexes by fixing:

$$
\begin{gathered}
L[n]=0 \forall n \geq 0 \\
C[0]=C_{M A X}
\end{gathered}
$$

Then, equation ( 55 ) becomes: 


$$
\Delta C[n]=-K_{r} C[n] \Delta t
$$

thus, the higher values for $K_{r}$, the faster the ligands unbind from the receptor. Due to its low $K_{r}, \alpha$-adrenergic receptors are not able to fulfill the minimum requirements. Even for very low frequencies (several $\mathrm{Hz}$ ), these receptors offer output amplitudes below 0.1 molecules. In other words, the receptor would not detect any molecule for the variation of the input sinus in $L(t)$.

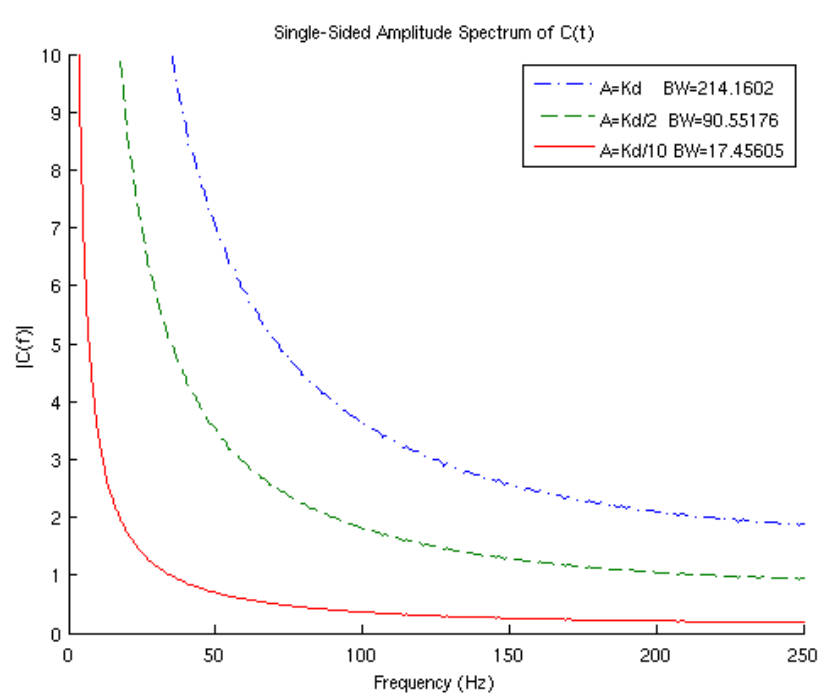

FIG. 32 BANDWIDTH FOR AN IL RECEPTOR AND DIFFERENT AMPLITUDES OF THE SINUS LIGAND SIGNAL.

However, the use of the light chain interleukin (IL) as a receptor highly improves the system performance. As it is shown in Fig. 33, the frequency response of IL turns this cell to be the main candidate for being implemented in the capillary circuit. We define the molecular bandwidth (BW) as the maximum frequency in the input signal that provokes 2 receptors to change in the IL cell. As shown in Fig. 32, the higher molecular BW that can be achieved is $214.16 \mathrm{~Hz}$ (for a ligand amplitude of $K d$ ). Reducing the input amplitude also reduces the molecular bandwidth. Hence, for a sinus amplitude of $0.5 \mathrm{Kd}$, we obtain a BW of $90.55 \mathrm{~Hz}$. For an amplitude $A=0.1 K d$, the maximum reachable bandwidth is found to be $14.45 \mathrm{~Hz}$. 

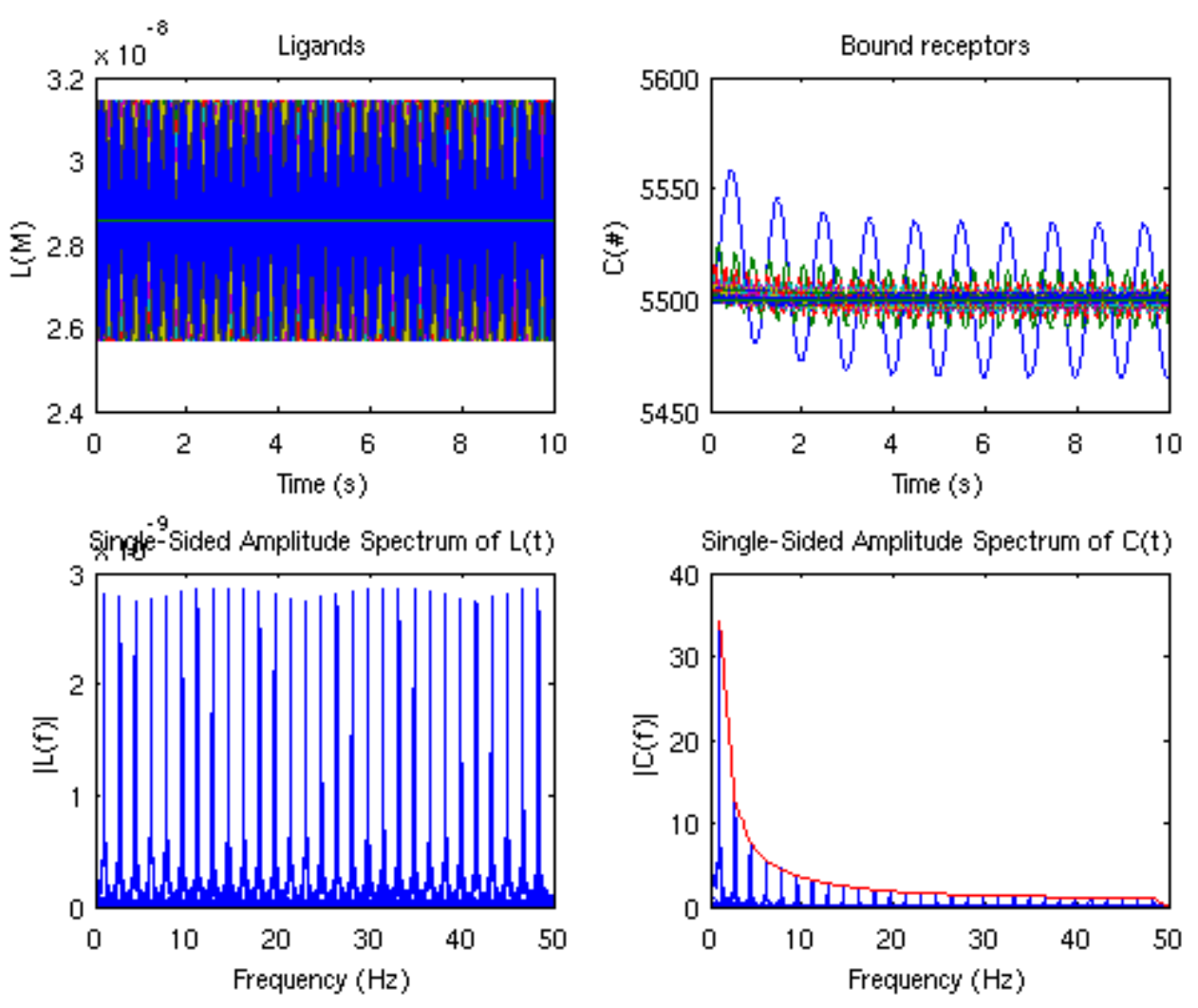

FIG. 33 LIGHT INTERLEUKIN RECEPTOR SIMULATION. TOP-LEFT: INPUT SIGNAL AS A SINUSOID VARIATION IN LIGAND CONCENTRATION. TOP-RIGHT: NUMBER OF LIGANDS THAT HAVE BEEN CAPTURED BY RECEPTORS. BOTTOM-LEFT: SPECTRAL ANALYSIS OF INPUT SIGNALS (LIGANDS). BOTTOM-RIGHT: SPECTRAL ANALYSIS FOR BOUND RECEPTORS (COMPLEXES) 


\section{QUALITATIVE EVALUATION}

In this section, the aforementioned techniques are compared qualitatively to demonstrate the strengths and weaknesses of each option. We have to take into account that the parameters evaluation is explorative and meaningful only for general overview. In addition to this, wired and wireless options should not be wrapped in the same consideration parameters without having in mind that their different medium transmission makes them suitable for some applications and not for others.

First of all, hardware complexity is a parameter that should be considered. On the one hand, wireless options are more complex in emitter and receptor implementation. For instance, to mimic pheromones receptor we need an antenna with 3 filters and binding proteins in an aqueous medium (corresponding to second filter where hydrophobic components are discarded) [43]. On the other hand, the distance between emitter and receiver does not need additional hardware in wireless cases.

The second parameter considered is the diversity, which can boost throughput or be a key factor in channel definition. Pheromones, pollen, spores and capillaries (using hormones in capillaries flow circuit) provide a high diversity. Although light transduction option does not provide molecular diversity, frequency spectrum can be used to multiplex several signals. Axons option is an alternative with only one signal type to communicate with (action potential).

Regarding distance coverage, light transduction option is capable to reach the longest nodes, as its electromagnetic propagation enables the use of already existing optical wireless techniques, or even the use of optical fibers in the transmission path. The axons are also a very good alternative to reach maximum distances of a meter or several meters, using chemical synapse reactions to join more than one axon. Capillaries could be used to reach approximately the same range. On the other hand, pheromones and pollen or spores are the options with less range, being able to effectively communicate in centimeters scale. As [7] states, pheromonal communication in nature involving ranges from hundreds of meters are only feasible taking the advantage of the wind as the transporting medium.

In signal speed terms, the axons are the best option, being able to transport the impulse up to $90 \mathrm{~m} / \mathrm{s}$. However, the synapse between neurons dramatically reduces this velocity. Light option is also a very fast option, but the translation between molecules and light reduces transmission speed. In the case of capillaries, the speed is greatly affected by the fluid current 
(probably plasma), where the pump mechanism plays a key role. Pheromones and pollen propagate by diffusion means, and are slower than other methods. For example, in the diffusion of the attractant alarm substance of a worker ant, it is need around a minute to reach $15 \mathrm{~cm}(0.0025 \mathrm{~m} / \mathrm{s})[7]$.

The reliability is another fundamental parameter. Wired options have in this case higher scores, being axons the most reliable option. In axons case the connection is peer to peer, whereas in capillaries the particles are taken from the flow current circulating through its pipes. Light transduction is also a very reliable option. In pollen, spores and pheromones it could be of interest to include error correction or cyclic codes to add robustness to the transmission.

Finally, the noise would affect more pollen and pheromones than the other options. In diffusion methods the presence of wind, other particles and possible chemical recombination should be considered as noise sources. In light option, the electromagnetic interference (caused by other EM signals) and the translation between molecular and optical signal should be considered as the main noise challenges. In the axons case, the presence of noise would not be very relevant, as myelin isolation would protect the signal from external interferences. In capillaries, the concrete receiver that binds the particle (hormone) should be modeled to determine the noise parameter (for example the probability of binding not desired particles and the probability of binding failure for the desired molecule). 


\section{CONCLUSION}

Nanotechnology is a novel field exponentially acquiring attention from the research community in the last years. The development of nano-machines following the bio-inspired approach, which is based on the usage of already existing biological elements as building blocks, offers promising solutions. However, very little empirical work has been done. Despite of some recent short range nano-communication experiments (e.g., calcium signaling in [53] or molecular motors in [24]), only theoretical simulations can be found. Hence, the real availableness of proposed communication methods has to be tested in future phases of nanoresearch roadmap.

Long-range nano-communication is an unexplored research area, capable of providing unique features for specific applications. Semiconductor or short-range molecular nano-comunication techniques cannot provide the properties that long-range applications require. When dealing with distances from a few centimeters to several meters, it is demanded new solutions for the improvement of the signal speed, reliability, energy consumption and hardware requirements.

In this paper several options have been proposed to communicate nodes at nano scale covering distances from few centimeters to several meters. Pheromones, pollen, spores, light transduction, capillaries and axons are analyzed and compared. However, it should be taken into account that each implementation could be more suitable depending on the concrete application, the network topology or the network environment.

Nevertheless, light transduction -converting molecular signal into optical information- is the most promising technique. As micro and nano elements will coexist in first nanotechnology stages, this option will act as an interface to interconnect different networks. The optical bridge between two molecular nanonetworks is also possible with this option.

The modeling of the long range options proposed in this paper should be imperative to analyze which of them offer better features. The information throughput, robustness or external factors influence in each option is the next step in that nanonetworking scope. Modeling, simulations, empirical results and detailed physical description should be done before going any further. Rebuilding upper layers in the protocol stack only make sense when first layer is reliable and have been tested or exhaustively simulated. 


\section{REFERENCES}

[1] I. Akyildiz, F. Brunetti, and C. Blazquez, "NanoNetworking: A New Communication Paradigm", Computer Networks Journal (Elsevier), Vol. 52, pp. 2260-2279, August, 2008.

[2] C.N. Allen, G. Ortner, C. Dion, P.J. Poole et al, "External-cavity quantum-dot laser tunable through 1.55 um", Applied Physics Letters, 2006.

[3] L. Alonso-Nanclares, J. Gonzalez-Soriano et al, "Gender differences in human cortical synaptic density", Proceedings of the National Academy of Sciences, 2008.

[4] A.J. Bateman, "Contamination of seed crops", Heredity, 1947.

[5] J.B. Baxter and E.S. Aydil, "Nanowire-based dye-sensitized solar cells", Applied Physics Letters, 2005.

[6] M. Born, E. Wolf, A.B. Bhatia, "Principles of optics", 7th expanded ed., Cambridge Univeristy Press, 1999.

[7] W. H. Bossert and E. Wilson, "Analysis of Olfactory Communication among Animals", Journal of Theoretical Biology, pp.443-469, 1963.

[8] J.P. Brody, P. Yager, "Low Reynolds number micro-fluidic devices", Technical Digest. Solid State Sensors and Actuator Workshop, 1996.

[9] L. Brus, "Chemistry and Physics of Semiconductor Nanocrystals", The 37th Middle Atlantic Regional Meeting, 2005.

[10] J.A. Byers, "Active space of pheromone plume and its relationship to effective attraction radius in applied models", Journal of Chemical Ecology, 2008.

[11] J.A. Byers, "Interactions of pheromone component odor plumes of western pine beetle", Journal of Chemical Ecology, 1987.

[12] D.Y. Chen, N.J. Dovichi, "Single-Molecule Detection in Capillary Electrophoresis: Molecular Shot Noise as a Fundamental Limit to Chemical Analysis", Glycobiology, 1994.

[13] S. Crosson and K. Moffat, "Photoexcited Structure of a Plant Photoreceptor Domain Reveals a Light-Driven Molecular Switch", The plant cell, 2002.

[14] M. Dai, H. E. Fisher et al, "The creation of a novel fluorescent protein by guided consensus engineering", Protein Engineering Design and Selection, Oxford University Press, 2007.

[15] P.K. Dasgupta and S. Liu, "Electroosmosis: A Reliable Fluid Propulsion System for Flow Injection Analysis", Analytical Chemistry, 1994.

[16]A. De, S.S. Gambhir, "Noninvasive imaging of protein-protein interactions from live cells and living subjects using bioluminescence resonance energy transfer" , The FASEB Journal, 2005.

[17] A.M. Derfus, WCW Chan et al, "Probing the Cytotoxicity of Semiconductor Quantum Dots", Nano letters, 2004.

[18]A Destexhe, ZF Mainen, TJ Sejnowski, "An efficient method for computing synaptic conductances based on a kinetic model of receptor binding", Neural Computation, 1994.

[19] A.M. DeWitt, K. Andersson, G. Peltre, J. Lidholm, "Cloning, expression and immunological characterization of full-length timothy grass pollen allergen $\mathrm{Phl} p 4$, a berberine bridge enzyme-like protein with homology to celery allergen Api g 5", Clinical \& Experimental Allergy, 2006.

[20]J.S. Dittman, A.C. Kreitzer, W.G. Regehr, "Interplay between facilitation, depression, and residual calcium at three presynaptic terminals", Journal of Neuroscience, 2000.

[21] K.E. Drexler, "Molecular nanomachines: physical principles and implementation strategies", Annual Review of Biophysics and Biomolecular Structure, 1994.

[22] J.S. Elkinton, R.T. Cardé, C.J. Mason, "Evaluation of time-average dispersion models for estimating pheromone concentration in a deciduous forest", Journal of Chemical Ecology, 1984. [23] Encyclopædia Britannica 2008, "axon", Retrieved December 10, 2008, from Encyclopædia Britannica Online: http://www.britannica.com/EBchecked/topic/46342/axon 
[24] A. Enomoto, M. Moore, T. Nakano et al., "A molecular communication system using a network of cytoskeletal filaments", Proceedings of the 2006 NSTI Nanotechnology Conference, May 2006.

[25]B. Ermentrout, "The Analysis of Synaptically Generated Traveling Waves", Journal of Computational Neuroscience 5, 1998.

[26]H. Fan, E.W. Leve, C. Scullin, J. Gabaldon et al , "Surfactant-assisted synthesis of water-soluble and biocompatible semiconductor quantum dot micelles" , Nano Letters, 2005.

[27]X. Gao, L Yang, JA Petros et al, "In vivo molecular and cellular imaging with quantum dots", Current Opinion in Biotechnology, 2005.

[28] G.D. Giddings, N.R.S. Hamilton, M.D. Hayward, "The release of genetically modified grasses. Part 2: the influence of wind direction on pollen ", Theoretical and Applied Genetics, 1997.

[29] J.W. Goodman, "Introduction to Fourier optics", 3rd ed., Roberts \& Company Publishers, 2004.

[30] P.H. Gregory, "The dispersion of air-borne spores", Transactions of the British Mycological Society, 1945.

[31] M.A. Haidekker, A.G. Tsai, T. Brady, H.Y. Stevens et al, "A novel approach to blood plasma viscosity measurement using fluorescent molecular rotors", American Journal of Physiology, 2002.

[32] G. Hanson, "Fundamental transmitting properties of carbon nanotube antennas," Antennas and Propagation, IEEE Transactions on, vol. 53, no. 11, pp. 3426-3435, Nov. 2005.

[33] R. Hardman, "A toxicologic review of quantum dots: toxicity depends on physicochemical and environmental factors", Environmental health perspectives, 2006.

[34] A. Hoshino, K. Fujioka, T. Oku et al, "Physicochemical properties and cellular toxicity of nanocrystal quantum dots depend on their surface modification", Nano Letters, 2004.

[35] J.B. Hursh, "Conduction velocity and diameter of nerve fibers", American Journal of Physiology, 1939.

[36] N. Jarosz, B. Loubet, L. Huber, "Modelling airborne concentration and deposition rate of maize pollen", Atmospheric Environment, 2004.

[37] J.M. Jornet Montana, "A Graphene Nanoribbon Antenna for Electromagnetic Nanocommunications", to appear.

[38] A. Kuparinen, "Mechanistic models for wind dispersal", TRENDS in Plant Science, 2006.

[39] J. Kawaoka and F.A. Gomez, "Use of mobility ratios to estimate binding constants of ligands to proteins in affinity capillary electrophoresis", Journal of Chromatography B, 1998.

[40] S.K. Kufer, E.M Puchner, H. Gumpp, T. Liedl, H.E. Gaub, "Single-molecule cut-and-paste surface assembly", Science, 2008.

[41] M. Law, L.E. Greene, J.C. Johnson, R. Saykally, P. Yang, "Nanowire dye-sensitized solar cells", Nature Materials, 2005.

[42] J. Law, F. Regnier, "Pheromones", in Annual Review of Biochemistry vol. 40, July 1971.

[43] W. Leal, "Pheromone Reception", in Topics in Current Chemistry, Berlin Heidelberg, Springer-Verlag, 2005.

[44]J.S. Liaw and T.W. Berger, "Dynamic synapse: a new concept of neural representation and computation", Hippocampus, 1996.

[45]J.J. Linderman, P.A. Mahama, K.E. Forsten and D.A. Lauffenburger, "Diffusion and Probability in Receptor Binding and Signaling", Advances in Chemical Engineering, 1994.

[46] H.Y. Liu, S.L. Liew, T. Badcock, D.J. Mowbray et al, "p-doped $1.3 \mu \mathrm{m}$ InAs/ GaAs quantum-dot laser with a low threshold current density and high differential efficiency", Applied Physics Letters, 2006. 
[47]B. Lu, W. M. Yamada, and T. W. Berger, "Nonlinear High-Order Model for Dynamic Synapse with Multiple Vesicle Pools", Neurodynamics of Cognition and Consciousness, 2007.

[48]A. Manwani, C. Koch, "Synaptic transmission: An information-theoretic perspective", Advances in Neural Information Processing Systems 10, 1997.

[49] S. Matsunaga, K. Schutze, I.S. Donnison et al. "Single pollen typing combined with laser-mediated manipulation", The Plant Journal, 1999.

[50]X. Michalet, F.F. Pinaud, L.A. Bentolila, J.M. Tsay et al, "Quantum dots for live cells, in vivo imaging, and diagnostics", Science, 2005.

[51] A. Miyawaki, J. Llopis et al, "Fluorescent indicators for $\mathrm{Ca} 2+$ based on green fluorescent proteins and calmodulin.", Nature, 1997.

[52] M. Moore, A. Enomoto, T. Nakano et al, "A Design of a Molecular Communication System for Nanomachines Using Molecular Motors", Pervasive Computing and Communications Workshops, 2006.

[53] T. Nakano, Y.H. Hsu, W.C. Tang, T. Suda et al, "Microplatform for intercellular communication", Nano/Micro Engineered and Molecular Systems, 2008.

[54] T. Nakano, T. Suda, M. Moore et al, "'Molecular communication for nanomachines using intercellular calcium signaling", 5th IEEE Conference on Nanotechnology, 2005.

[55] R. Nathan, U. N. Safriel and I. Noy-Meir, "Field validation and sensitivity analysis of a mechanistic model for tree seed dispersal by wind", Ecology, 2001.

[56] C.R. Nave. "HyperPhysics". Georgia State University, 2005. http://hyperphysics.phy-astr.gsu.edu/hbase/biology/actpot.html

[57] D.L. Nelson, M.M. Cox, "Principles of biochemistry" 5th ed., Freeman, 2008.

[58] M.A. Nielsen, I. Chuang, L.K. Grover, "Quantum computation and quantum information", American Journal of Physics, 2002.

[59] Ohio University, "Scientists find flaw in quantum dot construction", Physorg 2005. http://www.physorg.com/news3009.html

[60] G. Patterson, R.N. Day, D. Piston, "Fluorescent protein spectra", Journal of Cell Science, 2001

[61] J. Philibert, "One and a half century of diffusion: Fick, Einstein, before and beyond," Diffusion Fundamentals, vol. 2, pp. 1.1-1.10, 2005.

[62]M. Pierobon and I. Akyildiz, "A Physical Channel Model for Molecular Communication in Nanonetworks", submitted for journal publication March 2009.

[63] N. Roca Lacasa, "Modeling the Molecular Communication Nanonetworks", Universitat Politècnica de Catalunya, January 2009.

[64] O. Sallent, J.L. Valenzuela, R. Agustí, "Principios de comunicaciones móviles", Ediciones UPC, 2003.

[65] C. Saito et al, "Angiosperm species that produce sperm cell pairs or generative cells with polarized distribution of DNA-containing organelles", Sexual Plant Reproduction, 2002.

[66] D. P. Sierra, N. A. Weir, J. F. Jones, "A Review of Research in the Field of Nanorobotics", Sandia Report, Office of Scientific and Technical Information, US Department of Energy, October. 2005.

[67]M.K. So, C. Xu, A.M. Loening, S.S. Gambhir, J. Rao, "Self-illuminating quantum dot conjugates for in vivo imaging", Nature biotechnology, 2006.

[68]M.K. So, C. Xu, A.M. Loening, S.S. Gambhir, J. Rao, "Self-illuminating quantum dot conjugates for in vivo imaging: Supplementary Notes", http://www.nature.com/nbt/journal/v24/n3/suppinfo/nbt1188_S1.html

[69]M.B. Soons, G.W. Heil, R. Nathan, G.G. Katul, "Determinants of long-distance seed dispersal by wind in grasslands", Ecology, 2004. 
[70] T. Suda, M. Moore, T. Nakano, R. Egashira and A. Enomoto, "Exploratory Research on Molecular Communication between Nanomachines" in Genetic and Evolutionary Computation Conference, Washington DC, June 2005.

[71] O.G. Sutton, "Micrometeorology", McGraw-Hill, 1953.

[72]R. Swaminathan, C.P. Hoang, A.S. Verkman, "Photobleaching Recovery and Anisotropy Decay of Green Fluorescent Protein GFP-S65T in Solution and Cells: Cytoplasmic Viscosity Probed by Green Fluorescent Protein Translational and Rotational Diffusion", Biophysical journal, 1997.

[73] J.D. Tice, H. Song, A.D. Lyon, R.F. Ismagilov, "Formation of droplets and mixing in multiphase microfluidics at low values of the Reynolds and the the Capillary Numbers", American Chemical Society, 2003.

[74] University of New Jersey and University of California San Diego, "RCSB Protein Data Bank", http://www.rcsb.org/pdb

[75]E.B. Van Munster, G.J. Kremers, M.J.W. Adjobo-Hermans, T.W.J. Gadella Jr., "Fluorescence resonance energy transfer (FRET) measurement by gradual acceptor photobleaching", Journal of Microscopy, 2005.

[76] R.W. Wagner and J.S. Lindsey, "A molecular photonic wire", Journal of the American Chemical Society, 1994.

[77] J.D. Wilson, T.K. Flesch, "Trajectory curvature as a selection criterion for valid lagrangian stochastic dispersion models", Boundary-Layer Meteorology, 1997.

[78] J. Yu, J. Wang, S. Lou, T. Wang, Y. Jiang, "Small molecular and polymer organic light-emitting diodes based on novel iridium complex phosphor", Displays, 2008.

[79] L.X. Yu, J. Nasrallah, R. Valenta, M.V. Parthasarathy, "Molecular cloning and mRNA localization of tomato pollen profilin", Plant Molecular Biology, 1998.

[80] P Zhao, C Fang, C Xia, D Liu, S Xie, "15, 16-Dinitrile DDP/CPD as a possible solid-state optical molecular switch", Chemical Physics Letters, 2008. 